\title{
Mean Square Error and Limit Theorem for the Modified Leland Hedging Strategy with a Constant Transaction Costs Coefficient
}

\author{
by Sébastien Darses* ${ }^{*}$ Emmanuel Denis ${ }^{\dagger}$ \\ Université Aix-Marseille I and Université Paris Dauphine
}

Keywords: Asymptotic hedging - Leland-Lott strategy - Transaction costs Martingale limit theorem.

\section{INTRODUCTION}

The present paper is concerned with the study of asymptotic hedging in the presence of transaction costs. The asymptotic replication of a given payoff is performed via a modified Leland's strategy recently introduced in [2].

Let us briefly recall the history and the main known results about Leland's strategy. In 1985 Leland suggested an approach to price contingent claims under proportional transaction costs. His main idea was to use the classical Black-Scholes formula with a suitably adjusted volatility for a periodically revised portfolio whose terminal value approximates the payoff. The intuition behind this practical method is to compensate for transaction cost by increasing the volatility in the following way:

$$
\widehat{\sigma}_{t}^{2}=\sigma^{2}+\sigma \sqrt{n} k_{n} \sqrt{8 / \pi} \sqrt{f^{\prime}(t)},
$$

where $n$ is the number of the portfolio revision dates and $k_{n}=k_{0} n^{-\alpha}, \alpha \in\left[0, \frac{1}{2}\right]$ is the transaction costs coefficient generally depending of $n ; f$ is an increasing and smooth function whose inverse $g:=f^{-1}$ defines the revision dates $t_{i}^{n}:=g\left(\frac{i}{n}\right)$, $1 \leq i \leq n$.

The principal results on convergence for models with transaction costs can be described as follows. First consider the case of approximate hedging of the European

${ }^{*}$ LATP, Université Aix-Marseille I, France. Email: darses@cmi.univ-mrs.fr

${ }^{\dagger}$ Cérémade, Paris Dauphine University, France. Email : emmanuel.denis@ceremade.dauphine.fr

The authors thank the "Chair Les Particuliers Face aux Risque" sponsored by Groupama for their support. 
call option using the strategy with periodic portfolio revisions (i.e $g(t)=t$ ). We know the following results with $T=1$ :

(a) For $\alpha=\frac{1}{2}$, Lott gave the first rigorous result on the convergence of the approximating portfolio value $V_{1}^{n}$ to the payoff $V_{1}=\left(S_{1}-K\right)_{+}$. The sequence $V_{1}^{n}-V_{1}$ tends to zero in probability [10], and a stronger result holds: $n \mathbf{E}\left(V_{1}^{n}-V_{1}\right)^{2}$ converges to a constant $A_{1}>0$ [5];

(b) For $\alpha \in\left(0, \frac{1}{2}\right)$, the sequence $V_{1}^{n}-V_{1}$ tends to zero in probability (see [8]), and it is shown in [1] that $n^{p_{\alpha}} \mathbf{E}\left(V_{1}^{n}-V_{1}\right)^{2} \rightarrow 0$ as $n \rightarrow \infty$, with $p_{\alpha}<\alpha$.

(c) For $\alpha=0$, the terminal values of portfolios do not converge to the European call as shown by Kabanov and Safarian [8]. Namely, there is a negative $\sigma\left\{S_{1}\right\}$-measurable random variable $\xi$ such that $V_{1}^{n}-V_{1} \rightarrow \xi$ in probability. Pergamenshchikov [11] then analyzed the rate of convergence and proved a limit theorem: the sequence $n^{\frac{1}{4}}\left(V_{1}^{n}-V_{1}-\xi\right)$ converges in law to a mixture of Gaussian distributions [11]. He noticed that one can increase the modified volatility to obtain the asymptotic replication. To do so, he utilizes the explicit form of the systematic hedging error for the European call option. For related results see also [6] and [12].

For models including uniform and non-uniform revision intervals one needs to impose certain conditions on the scale transform $g$. Generalizations of some of the above results to this more technical case as well as extensions to contingent claims of the form $h\left(S_{1}\right)$ can be found in $[12,5,1]$. In particular, $n^{1 / 2+\alpha} \mathbf{E}\left(V_{1}^{n}-V_{1}\right)^{2}$ converges to a constant in the case $\alpha>0$. Moreover, for $\alpha=\frac{1}{2}$, the distributions of the process $Y_{t}^{n}:=n^{\frac{1}{2}}\left(V_{t}^{n}-\widehat{V}_{t}\right)^{\ddagger}$ in the Skorohod space $\mathcal{D}[0,1]$ converges weakly to the distribution of a two-dimensional Markov diffusion process component (see [4]). Notice that the asymptotic replication still does not hold for $\alpha=0$ in this more general setting. For more details we refer to $[1,3,4]$ and references therein.

We solve the case $\alpha=0$ for a large class of payoff and with specific non uniform revision dates by means of the modified strategy introduced in [2]. This one makes the portfolio's terminal value converge to the contingent claim as $n$ tends to infinity, that is the approximation error vanishes. The analysis we performed here suggests that it might be difficult to obtain a better convergence rate regarding uniform

\footnotetext{
${ }^{\ddagger}$ Note that $Y_{t}^{n}$ corresponds to the deviation (up to a multiplicative constant) between the "real world" portfolio and the theoretical Leland's portfolio $\widehat{V}_{t}=\widehat{C}\left(t, S_{t}\right)$ where $\widehat{C}$ is the modified heat equation solution suggested by Leland whose terminal value is the payoff function.
} 
revision dates. In the framework of the non uniform grid we use, concentrating the revision dates near the maturity $T=1$ accelerates the convergence rate. We leave the issue concerning periodic revision dates as an open problem.

The asymptotic behavior of the hedging error is a practical important issue. Since traders obviously prefer gains than losses, measuring the $L^{2}$-norm of hedging errors is strongly criticized. Of course, the limiting distribution of the hedging error is much more informative. Our present work also aims at tackling this issue: we prove that

$$
n^{\frac{1}{4}+p}\left(V_{1}^{n}-h\left(S_{1}\right)\right) \underset{n \rightarrow \infty}{\stackrel{d}{\longrightarrow}} Z,
$$

where the law of $Z$ is explicitly identified and $p>0$ depends on the choosen grid.

The paper is organized as follows. In Section 2, we introduce the basic notations, models and assumptions of our study; In particular we recall the modified Leland's strategy defined in [2]. In Section 3, we state our main result: a limit theorem for the renormalized asymptotic hedging error. In Section 4, we establish two lemmas concerning, on one hand, random variables constructed from the geometric Brownian motion, and on the other hand, some change of variables for the revision dates. These auxiliary results will be used repeatedly throughout the paper. In Section 5, we prove the main result. An appendix finally recalls all the known technical results we need for the various proofs.

\section{Notations ANd Models}

2.1. Black-Scholes model and hedging strategy. We are given a filtered probability space $\left(\Omega, \mathcal{F},\left(\mathcal{F}_{t}\right)_{[0,1]}, \mathbb{P}\right)$ on which a standard one-dimensional $\left(\mathcal{F}_{t}\right)$-adapted Brownian motion $W$ is defined. As usual, we denote by $L^{2}(\Omega)$ the space of square integrable $\mathcal{F}_{1}$-measurable random variables endowed with its norm $\|X\|_{2}:=\sqrt{\mathbf{E} X^{2}}$.

We consider the classical Black-Scholes model composed of two assets without transaction costs, i.e. $k_{0}=0$ and $\widehat{\sigma}=\sigma$. The first one is riskless (bond) with the interest rate $r=0$ and the second asset is $S=\left(S_{t}\right), t \in[0,1]$, a geometric Brownian motion that is

$$
S_{t}=S_{0} e^{\sigma W_{t}-\frac{1}{2} \sigma^{2} t}
$$

It satisfies the SDE

$$
d S_{t}=\sigma S_{t} d W_{t}
$$


with positive constants $S_{0}, \sigma$. It means that the risky asset is seen under the martingale measure.

The well-known Black and Scholes problem without transaction costs is to hedge a payoff $h\left(S_{1}\right), h$ being a continuous function of polynomial growth. The pricing function solves the terminal valued Cauchy problem

$$
\left\{\begin{aligned}
C_{t}(t, x)+\frac{\sigma_{t}^{2}}{2} x^{2} C_{x x}(t, x) & =0, \quad t \in[0,1], x>0, \\
C(1, x) & =h(x) .
\end{aligned}\right.
$$

Its solution can be written as

$$
C(t, x)=\int_{-\infty}^{\infty} h\left(x e^{\rho_{t} y-\frac{\rho_{t}^{2}}{2}}\right) \varphi(y) d y
$$

where $\rho_{t}^{2}=(1-t) \sigma^{2}$ and $\varphi$ is the standard Gaussian density.

Without transaction costs $(\sigma=\widehat{\sigma})$ the self-financed portfolio process reads

$$
V_{t}=C\left(0, S_{0}\right)+\int_{0}^{t} C_{x}\left(u, S_{u}\right) d S_{u} .
$$

In the Itô formula for $C\left(t, S_{t}\right)$ the integral over $d t$ vanishes and, therefore, $V_{t}=$ $C\left(t, S_{t}\right)$ for all $t \in[0,1]$. In particular, $V_{1}=h\left(S_{1}\right)$ : At maturity the portfolio $V$ replicates the terminal payoff of the option. Modeling assumptions of the above formulation include frictionless market and continuous trading for instance.

However, an investor revises the portfolio at a finite set of dates

$$
\mathcal{T}^{n}=\left\{t_{i} \in[0,1], i=0, \cdots, n\right\}
$$

and keeps $C_{x}\left(t_{i}, S_{t_{i}}\right)$ units of the stock until the next revision date $t_{i+1}$. It is well known that this discretized model converges to the Black-Scholes one in the sense that the corresponding portfolio terminal value converges to the payoff as the number of revision dates tends to infinity.

2.2. Reminder about Leland's strategy. We are now concerned with transaction costs. We directly work in a discrete time setting.

Leland suggested to replace $\sigma$ in the Cauchy problem above by a suitable modified volatility $\widehat{\sigma}$. In the case where $\widehat{\sigma}$ does not depend on $t$, the solution $\widehat{C}$ satisfies

$$
\widehat{C}(t, x)=C(t, x, \widehat{\sigma})
$$

i.e. practitioners do not need to rectify their algorithms to compute the strategy. Leland obtained an explicit expression of $\widehat{\sigma}$ by equalizing the transaction costs of the portfolio and the drift term generated by the additional term $\widehat{\sigma}-\sigma^{2}>0$ in 
the Ito expansion of the payoff $h\left(S_{1}\right)=\widehat{C}\left(1, S_{1}\right)$. In the general case, the pricing function can be written as

$$
\widehat{C}(t, x)=\int_{-\infty}^{\infty} h\left(x e^{\rho_{t}^{n} y-\left(\rho_{t}^{n}\right)^{2} / 2}\right) \varphi(y) d y
$$

where

$$
\begin{aligned}
\left(\rho_{t}^{n}\right)^{2} & :=\int_{t}^{1} \widehat{\sigma}_{s}^{2} d s, \\
\widehat{\sigma}_{t}^{2} & :=\sigma^{2}+\sigma \sqrt{n} k_{n} \sqrt{8 / \pi} \sqrt{f^{\prime}(t)}
\end{aligned}
$$

$\varphi$ is the Gaussian density and $g=f^{-1}$ is the revision date function.

2.3. A possible modification of Leland's strategy. In the model with proportional transaction costs and a finite number of revision dates the current value of the portfolio process at time $t$ is described as

$$
V_{t}^{n}:=V_{0}^{n}+\int_{0}^{t} D_{u}^{n} d S_{u}-\sum_{t_{i}<t} k_{0} S_{t_{i}}\left|D_{i+1}^{n}-D_{i}^{n}\right|
$$

where $D^{n}$ is a piecewise-constant process with $D^{n}=D_{i}^{n}$ on the interval $\left(t_{i-1}, t_{i}\right]$, $t_{i}=t_{i}^{n}, i \leq n$, are the revision dates, and $D_{i}^{n}$ are $\mathcal{F}_{t_{i-1}}$-measurable random variables.

Recall that the transaction costs coefficient is a constant $k_{0}>0$ (that is $\alpha=0$ in the Leland model) and the dates $t_{i}$ are defined by a function $g$, namely $t_{i}=g\left(\frac{i}{n}\right)$. Let us denote by $f$ the inverse of $g$. Set for all $i_{0}<n$

$$
J_{i_{0}}^{n}(t)=\left\{i \geq i_{0}, t_{i} \leq t, t_{i} \in \mathcal{T}^{n}\right\}
$$

and let us define the dates

$$
\begin{aligned}
& t_{n}^{-}(t)=t_{(n-1) \wedge \max J_{0}^{n}(t)} \\
& t_{n}^{+}(t)=t_{1+(n-1) \wedge \max J_{0}^{n}(t) .}
\end{aligned}
$$

The "enlarged volatility", depending on $n$, is given by the formula (2.9).

We modify the usual Leland strategy (see [2]) by considering the process $D^{n}$ with

$$
D_{i}^{n}:=\widehat{C}_{x}\left(t_{i-1}, S_{t_{i}-1}\right)-\sum_{j=1}^{i-1} \int_{t_{j-1}}^{t_{j}} \widehat{C}_{x t}\left(u, S_{t_{j-1}}\right) d u .
$$

Moreover, let us define

$$
K_{t}^{n}:=\sum_{i \in J_{1}^{n}(t)} \Delta K_{t_{i}^{n}}^{n}
$$


where $\Delta K_{t_{0}^{n}}^{n}:=0$ and for $i \geq 1$,

$$
\Delta K_{t_{i}^{n}}^{n}:=-\int_{t_{i-1}}^{t_{i}} \widehat{C}_{x t}\left(u, S_{u}\right) d u .
$$

In the same way, we set

$$
L_{t}^{n}:=\sum_{i \in J_{1}^{n}(t)} \Delta L_{t_{i}^{n}}^{n}
$$

where $\Delta L_{t_{0}^{n}}^{n}:=0$ and for $i \geq 1$,

$$
\Delta L_{t_{i}^{n}}^{n}:=-\int_{t_{i-1}}^{t_{i}} \widehat{C}_{x t}\left(u, S_{t_{i-1}}\right) d u .
$$

2.4. Assumptions and notational conventions. Throughout the paper, we adopt the following rules:

(i) we will often omit the indexes $n$ and the variable $t$ (especially in the appendix) when there is no ambiguity;

(ii) the constants $C$ appearing in the various inequalities is independent of $n$ and may change from one line to the next;

(iii) we use the classical Landau notations $O$ and $o$. These quantities will be always deterministic.

We use the abbreviations

$$
\begin{aligned}
\delta_{t} & :=\widehat{C}_{x}\left(t, S_{t}\right), \\
\gamma_{t} & :=\widehat{C}_{x x}\left(t, S_{t}\right) .
\end{aligned}
$$

We denote by $\left(\delta_{t}^{n}\right)_{t}$ the process equal to $\delta_{t_{i}^{n}}$ on the interval $\left[t_{i}^{n}, t_{i+1}^{n}\right)$ and $\left(\gamma_{t}^{n}\right)_{t}$ is defined similarly. For an arbitrary process $H$, we set $\Delta H_{t_{i}}=H_{t_{i}}-H_{t_{i-1}}$.

We will work under the following assumptions:

(A1) The function $g$ has the following form:

$$
g(t)=1-(1-t)^{\mu}, \quad \mu \in\left(1, \frac{3+\sqrt{57}}{8}\right) .
$$

(A2) $h$ is a convex and continuous function on $[0, \infty)$ which is twice differentiable except the points $K_{1}<\cdots<K_{p}$ where $h^{\prime}$ and $h^{\prime \prime}$ admit right and left limits; $\left|h^{\prime \prime}(x)\right| \leq M x^{-\beta}$ for $x \geq K_{p}$ where $\beta \geq 3 / 2$.

Assumption (A1) is not too restrictive. A trader can in particular choose $\mu$ sufficiently close to 1 to balance its portfolio quasi periodically. However, as we will see, it is more preferable to increase $\mu$ to obtain a better rate of convergence. 


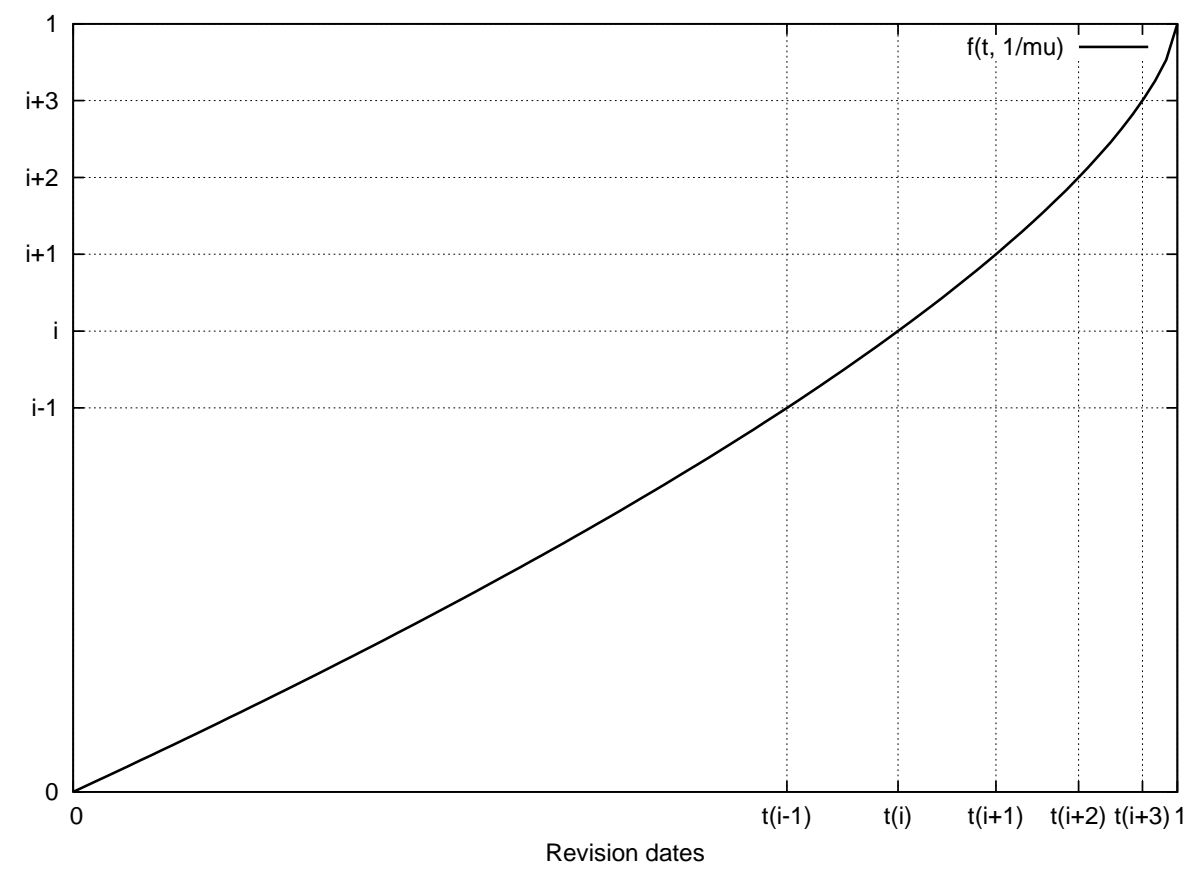

FIGURE 1. Revision dates with $f(t)=1-(1-t)^{\frac{1}{\mu}}, \mu=1.5$ and $n=10$.

Note that $f(t)=1-(1-t)^{1 / \mu}$, hence the derivative $f^{\prime}$ for $\mu>1$ explodes at the maturity date and so does the enlarged volatility. We define the increasing function

$$
p:=p(\mu):=\frac{\mu-1}{4(1+\mu)} .
$$

Under Assumption (A1), we have $0<p<1 / 16$.

In the sequel, the quantity

$$
\mathcal{Q}(\mu)=\frac{\mu^{1 / 2-2 p}(1+\mu)^{4 p}}{2^{4 p}(\sqrt{8 / \pi})^{4 p+1}}
$$

will frequently appear. 


\section{MAin Result}

In [2], it is proven that $V_{1}^{n}$ converges in probability to $h\left(S_{1}\right)$. Our main result here provides the rate of convergence and identifies the associated limit distribution of the deviation:

Theorem 3.1. Consider the portfolio $V^{n}$ defined by (2.10) and (2.11) under Assumptions (A1) and (A2). The following convergence then holds:

$$
n^{\frac{1}{4}+p}\left(V_{1}^{n}-h\left(S_{1}\right)\right) \underset{n \rightarrow \infty}{\stackrel{d}{\longrightarrow}} Z,
$$

where the law of $Z$ is given by the characteristic function $\phi_{Z}(s)=\mathbf{E} e^{-\frac{s^{2}}{2} \eta^{2}}$ with

$$
\begin{aligned}
\eta^{2}:=\mathcal{Q}(\mu)\left(k_{0} \sigma\right)^{1-4 p} S_{1}^{2} \times \\
\quad \int_{0}^{\infty} x^{4 p}\left\{\left(\int_{x}^{\infty} J\left(y, S_{1}\right) d y\right)^{2}+\left(1-\frac{2}{\pi}\right) \widetilde{J}\left(x, S_{1}\right)^{2}\right\} d x,
\end{aligned}
$$

and

$$
\begin{aligned}
& J\left(x, S_{1}\right):=\frac{1}{2 x} \int_{-\infty}^{\infty} h^{\prime}\left(S_{u} e^{\sqrt{x} y+x / 2}\right)\left(-y^{2}-\sqrt{x} y+1\right) \varphi(y) d y \\
& \widetilde{J}\left(x, S_{1}\right):=\frac{1}{\sqrt{x}} \int_{-\infty}^{\infty} h^{\prime}\left(S_{u} e^{\sqrt{x} y+x / 2}\right) y \varphi(y) d y .
\end{aligned}
$$

Moreover

$$
n^{\frac{1}{2}+2 p} \mathbf{E}\left(V_{1}^{n}-h\left(S_{1}\right)\right)^{2} \underset{n \rightarrow \infty}{\longrightarrow} \mathbf{E} \eta^{2} .
$$

\section{Auxiliary Results}

4.1. Geometric Brownian motion and related quantities. In the sequel, we shall use the decomposition given by Ito formula

$$
\widehat{C}_{x}\left(t, S_{t}\right)=\widehat{C}_{x}\left(0, S_{0}\right)+\widehat{M}_{t}^{n}+\widehat{A}_{t}^{n}
$$

where

$$
\begin{aligned}
\widehat{M}_{t}^{n} & :=\int_{0}^{t} \sigma_{u} S_{u} \widehat{C}_{x x}\left(u, S_{u}\right) d W_{u}, \\
\widehat{A}_{t}^{n} & :=\int_{0}^{t}\left[\widehat{C}_{x t}\left(u, S_{u}\right)+\frac{1}{2} \sigma_{u}^{2} S_{u}^{2} \widehat{C}_{x x x}\left(u, S_{u}\right)\right] d u .
\end{aligned}
$$

The process $\widehat{M}^{n}$ is a square integrable martingale on [0,1] by virtue of [2].

We set for $u<v$

$$
\mathcal{E}_{u}^{v}=\frac{S_{v}}{S_{u}}-1
$$


and

$$
\begin{aligned}
{\left[\mathcal{E}_{u}^{v}\right]_{c} } & =\mathbf{E}\left(\left|\mathcal{E}_{u}^{v}\right|\right)-\left|\mathcal{E}_{u}^{v}\right| . \\
\left\{\mathcal{E}_{u}^{v}\right\}_{s}^{2} & :=\left(\mathcal{E}_{u}^{v}\right)^{2} \operatorname{sgn} \mathcal{E}_{u}^{v} .
\end{aligned}
$$

In the sequel, we will use several times the following basic results.

Lemma 4.1. For all $i$ the following inequalities and expansions hold:

$$
\begin{aligned}
\mathbf{E}\left(\mathcal{E}_{u}^{v}\right)^{2 m} & \leq C_{m}(v-u)^{m}, \quad u \leq v \\
\mathbf{E}\left(\mathcal{E}_{t_{i-1}}^{t_{i}}\right)^{2} & =\sigma^{2} \Delta t_{i}(1+o(1)) \\
\mathbf{E}\left[\mathcal{E}_{t_{i-1}}^{t_{i}}\right]_{c}^{2} & =\left(1-\frac{2}{\pi}\right) \sigma^{2} \Delta t_{i}(1+o(1)) \\
\mathbf{E}\left[\mathcal{E}_{t_{i-1}}^{t_{i}}\right]_{c}^{2} \operatorname{sgn} \mathcal{E}_{u}^{v} & =\left(1-\frac{2}{\pi}\right) \sigma^{2}\left(\Delta t_{i}\right)^{\frac{3}{2}}(1+o(1)) \\
\mathbf{E}\left\{\mathcal{E}_{t_{i-1}}^{t_{i}}\right\}_{s}^{2} & =k\left(\Delta t_{i}\right)^{3 / 2}\left(1+o\left(n^{-1 / 4}\right)\right) .
\end{aligned}
$$

Proof. We refer to [1] or [4]. For the sake of completeness we recall the proof of the last one. Let us notice the equality in law

$$
\left\{\mathcal{E}_{t_{i-1}}^{t_{i}}\right\}_{s}^{2} \stackrel{d}{=}\left(\exp \left\{\sigma \sqrt{\Delta t_{j}} \xi-\sigma^{2} \Delta t_{j} / 2\right\}-1\right)^{2}\left(1_{\xi \geq \sigma \sqrt{\Delta t_{j}} / 2}-1_{\xi \leq \sigma \sqrt{\Delta t_{j}} / 2}\right)
$$

where $\xi$ is the standard Gaussian variable. Since $\xi$ and $-\xi$ has the same law, this yields

$$
\begin{aligned}
\mathbf{E}\left\{\mathcal{E}_{t_{i-1}}^{t_{i}}\right\}_{s}^{2}=\mathbf{E} & {\left[\left(e^{u \xi-u^{2} / 2}-1\right)^{2}-\left(e^{-u \xi-u^{2} / 2}-1\right)^{2}\right] 1_{\xi \geq u / 2} } \\
& -\mathbf{E}\left(e^{-u \xi-u / 2}-1\right)^{2} 1_{|\xi| \leq u / 2},
\end{aligned}
$$

where $u=\sigma \sqrt{\Delta t_{j}}$. Moreover, we have the inequality

$$
\mathbf{E}\left(e^{-u \xi-u^{2} / 2}-1\right)^{2} 1_{|\xi| \leq u / 2} \leq u^{4} .
$$

From [5], we recall that

$$
\mathbf{E}\left[\left(e^{u \xi-u^{2} / 2}-1\right)^{2}-\left(e^{-u \xi-u^{2} / 2}-1\right)^{2}\right] 1_{\xi \geq u / 2}=\frac{2}{\sqrt{2 \pi}} u^{3}+O\left(u^{4}\right) .
$$

We then conclude.

4.2. Basic results concerning the revision dates. The function $\rho_{t}$ decreases from $\rho_{0}$ to 0 . The following useful bounds are obvious:

$$
\begin{aligned}
& \rho_{t}^{2} \geq\left(\sigma^{2}+c n^{\frac{1}{2}}\right)(1-t) \\
& \rho_{t}^{2} \leq \sigma^{2}(1-t)+\sigma k_{0} n^{\frac{1}{2}} \sqrt{8 / \pi}(1-t)^{\frac{1}{2}}(1-f(t))^{\frac{1}{2}} .
\end{aligned}
$$


Moreover, it is straightforward that

$$
\rho_{t}^{2} \geq c n^{\frac{1}{2}} \sqrt{f^{\prime}(t)}(1-t)
$$

provided that $f^{\prime}$ is no decreasing.

Note that there is a constant $C$ independent of $n$ such that for all $i \leq n-1$,

$$
\frac{1-t_{i-1}}{1-t_{i}} \leq C
$$

From there we deduce

$$
\frac{\Delta t_{i}}{1-t_{i}} \leq C
$$

We shall often use the inequality

$$
\sum_{i=1}^{n-1} \frac{\Delta t_{i}}{1-t_{i}} \leq C \log (n)
$$

where $C$ is a constant independent of $n$.

Lemma 4.2. Fix $x>0$ and $t:=t(n, x)$ such that $x=\rho_{t}^{2}$. Set $x_{i-1}=\rho_{t_{i-1}}^{2}$ and $x_{i}=\rho_{t_{i}}^{2}$ where $t_{i-1}, t_{i}$ are such that $t \in\left[t_{i-1}, t_{i}\right)$. Then, $x \in\left(x_{i}, x_{i-1}\right]$ with $\left|x_{i-1}-x_{i}\right| \leq c n^{-1 / 2}, c$ is a constant. There exists a constant $C>0$ such that

$$
\frac{\Delta t_{i} n^{1 / 2+2 p}}{x_{i-1}-x_{i}} \leq C(x+1)
$$

Moreover, for a given $x,(1-t) \leq c n^{-1 / 2} x \rightarrow 0$ as $n \rightarrow \infty$ and

$$
\frac{\Delta t_{i} n^{1 / 2+2 p}}{x_{i-1}-x_{i}} \underset{n \rightarrow \infty}{\longrightarrow} \frac{\mathcal{Q}(\mu) x^{4 p}}{\left(\sigma k_{0}\right)^{4 p+1}} \text {. }
$$

Proof. Let us write

$$
\begin{aligned}
\frac{\Delta t_{i} n^{1 / 2+2 p}}{x_{i-1}-x_{i}} & =\frac{n^{2 p}}{\sigma^{2} n^{-1 / 2}+\sigma k_{0} \sqrt{8 / \pi} \frac{1}{\Delta t_{i}} \int_{t_{i-1}}^{t_{i}} \sqrt{f^{\prime}(u)} d u} \\
& \underset{n \rightarrow \infty}{\sim} \frac{n^{2 p}}{\sigma k_{0} \sqrt{8 / \pi} \sqrt{f^{\prime}\left(\tilde{t}_{i}\right)}}
\end{aligned}
$$

where $\tilde{t}_{i} \in\left[t_{i-1}, t_{i}\right]$. Moreover

$$
\begin{aligned}
x=\rho_{t}^{2} & =\sigma^{2}(1-t)+\sigma k_{0} \sqrt{8 / \pi} n^{1 / 2} \int_{t}^{1} \sqrt{f^{\prime}(u)} d u \\
& =\sigma^{2}(1-t)+\sigma k_{0} \sqrt{8 / \pi} n^{1 / 2} \frac{2 \mu^{1 / 2}}{1+\mu}(1-t)^{\frac{1+\mu}{2 \mu}}
\end{aligned}
$$

and

$$
1-t=\left(\frac{x-\sigma^{2}(1-t)}{\sigma k_{0} \sqrt{8 / \pi} n^{1 / 2}} \frac{1+\mu}{2 \mu^{1 / 2}}\right)^{\frac{2 \mu}{1+\mu}}
$$


Note that $x \geq c n^{1 / 2}(1-t)$ so that $(1-t) \leq c n^{-1 / 2} x \rightarrow 0$. In a similar way, we have

$$
x_{i-1}-x_{i}=\rho_{t_{i-1}}^{2}-\rho_{t_{i}}^{2}=\sigma^{2} \Delta t_{i}+c n^{1 / 2} \sqrt{f^{\prime}\left(\check{t}_{i}\right)} \Delta t_{i}
$$

where $\check{t}_{i} \in\left[t_{i-1}, t_{i}\right]$. We deduce that

$$
x_{i-1}-x_{i}=\sigma^{2} \Delta t_{i}+c n^{1 / 2} \sqrt{f^{\prime}\left(\check{t}_{i}\right)} g^{\prime}\left(\theta_{i}\right) n^{-1}
$$

where $\theta_{i} \in[(i-1) / n, i / n]$. Moreover,

$$
\sqrt{f^{\prime}\left(\check{t}_{i}\right)} g^{\prime}\left(\theta_{i}\right)=\frac{g^{\prime}\left(\theta_{i}\right)}{\sqrt{g^{\prime}\left(f\left(\check{t}_{i}\right)\right)}}
$$

is bounded since $f\left(\check{t}_{i}\right) \in[(i-1) / n, i / n], i \leq n-1$. Hence there is a constant $c$ satisfying $x_{i-1}-x_{i} \leq c n^{-1 / 2}$. Since $\rho$ is decreasing, $x \in\left[x_{i}, x_{i-1}\right]$.

Eventually, $\tilde{t}_{i} \in\left[t_{i-1}, t_{i}\right]$ is such that $\tilde{x}_{i}=\rho_{\tilde{t}_{i}}^{2} \in\left[x_{i}, x_{i-1}\right]$ and $\tilde{x}_{i} \rightarrow x$. Similarly we have

$$
1-\tilde{t}_{i}=\left(\frac{\tilde{x}_{i}-\sigma^{2}\left(1-\tilde{t}_{i}\right)}{\sigma k_{0} \sqrt{8 / \pi} n^{1 / 2}} \frac{1+\mu}{2 \mu^{1 / 2}}\right)^{\frac{2 \mu}{1+\mu}}
$$

which yields

$$
\sqrt{f^{\prime}\left(\tilde{t}_{i}\right)}=\mu^{-1 / 2}\left(\frac{\tilde{x}_{i}-\sigma^{2}\left(1-\tilde{t}_{i}\right)}{\sigma k_{0} \sqrt{8 / \pi} n^{1 / 2}} \frac{1+\mu}{2 \mu^{1 / 2}}\right)^{\frac{1-\mu}{1+\mu}}
$$

and

$$
\begin{aligned}
\frac{\Delta t_{i} n^{1 / 2+2 p}}{x_{i-1}-x_{i}} & \underset{n \rightarrow \infty}{\sim} \frac{n^{2 p}}{\sigma k_{0} \sqrt{8 / \pi} \sqrt{f^{\prime}\left(\tilde{t}_{i}\right)}} \\
& \underset{n \rightarrow \infty}{\sim} \frac{n^{2 p}}{\sigma k_{0} \sqrt{8 / \pi}} \mu^{1 / 2}\left(\frac{\sigma k_{0} \sqrt{8 / \pi} n^{1 / 2}}{\tilde{x}_{i}-\sigma^{2}\left(1-\tilde{t}_{i}\right)} \frac{2 \mu^{1 / 2}}{1+\mu}\right)^{\frac{1-\mu}{1+\mu}} \\
& \underset{n \rightarrow \infty}{\sim} \frac{1}{\sigma k_{0} \sqrt{8 / \pi}} \mu^{1 / 2}\left(\frac{\sigma k_{0} \sqrt{8 / \pi}}{\tilde{x}_{i}-\sigma^{2}\left(1-\tilde{t}_{i}\right)} \frac{2 \mu^{1 / 2}}{1+\mu}\right)^{\frac{1-\mu}{1+\mu}} .
\end{aligned}
$$

Since $\tilde{x}_{i} \rightarrow x$ and $\tilde{t}_{i} \rightarrow 0$, we deduce that

$$
\frac{\Delta t_{i} n^{1 / 2+2 p}}{x_{i-1}-x_{i}} \underset{n \rightarrow \infty}{\longrightarrow} \frac{1}{\sigma k_{0} \sqrt{8 / \pi}} \mu^{1 / 2}\left(\frac{\sigma k_{0} \sqrt{8 / \pi}}{x} \frac{2 \mu^{1 / 2}}{1+\mu}\right)^{\frac{1-\mu}{1+\mu}} .
$$

Since $0<(\mu-1) /(1+\mu)<1$, we also find a constant $c$ such that

$$
\frac{\Delta t_{i} n^{1 / 2+2 p}}{x_{i-1}-x_{i}} \leq c\left|\tilde{x}_{i}-\sigma^{2}\left(1-\tilde{t}_{i}\right)\right|^{\frac{\mu-1}{1+\mu}} \leq c(x+1),
$$

which concludes the proof.

We now stress an important remark regarding a slight abuse of notation repeatedly used along the paper. 
Remark 4.3. Throughout the sequel, we shall often use the change of variable $x=\rho_{t}^{2}$ with $d x=-\widehat{\sigma}_{t}^{2} d t$. For ease of notation, we will use the abuse of notation $t$ instead of $t(n, x):=\left(\rho^{2}\right)^{-1}(x)$ when applying this change of variable in an integral.

Similarly, a direct computation yields the following lemma.

Lemma 4.4. Set $y>0$ and $v:=v(n, y)$ such that $y=\rho_{v}^{2}$. There exists a constant $C>0$ such that

$$
\frac{(1-v) n^{1 / 2+2 p}}{y} \leq C y
$$

Moreover, for a given $y,(1-v) \leq c n^{-1 / 2} y \rightarrow 0$ as $n \rightarrow \infty$ and

$$
\frac{(1-v) n^{1 / 2+2 p}}{y} \underset{n \rightarrow \infty}{\longrightarrow} \frac{\mu^{-1 / 2-2 p}(1+\mu)^{4 p+1} y^{4 p}}{2^{4 p}\left(\sigma k_{0} \sqrt{8 / \pi}\right)^{4 p+1}} .
$$




\section{Proof of the Limit TheOREM}

The proof is divided into three parts. In Step 1 we split the hedging error into a martingale part $M$ and a residual part $\varepsilon$. In Step 2 we show that the residual terms tend to 0 in $L^{2}(\Omega)$ with rate $n^{\frac{1}{4}+p}$ as $n$ tends to infinity. We identify in Step 3 the asymptotic distribution of the martingale $n^{\frac{1}{4}+p} M^{n}$ and we conclude the proof of the main result.

5.1. Step 1: Splitting of the hedging error. Comparing Expression (2.10) with the Ito expansion of $h\left(S_{1}\right)=\widehat{C}\left(1, S_{1}\right)$ yields the following decompositions. The hedging error reads

$$
V_{1}^{n}-h\left(S_{1}\right)=M_{1}^{n}+\varepsilon_{1}^{n}
$$

where for all $n \in \mathbb{N}, M^{n}$ is a martingale of terminal value

$$
M_{1}^{n}:=k_{0} \sum_{i \leq n-1} \gamma_{t_{i-1}} S_{t_{i-1}}^{2}\left[\mathcal{E}_{t_{i-1}}^{t_{i}}\right]_{c}+\int_{0}^{1} K_{u}^{n} d S_{u} .
$$

The residual term can be splited as

$$
\varepsilon_{t}^{n}=R_{0}^{n}(t)+R_{1}^{n}(t)+R_{2}^{n}(t)+R_{3}^{n}(t)
$$

where

$$
\begin{aligned}
R_{0}^{n}(t) & :=k_{0} \sum_{i \in J_{1}^{n}(t)} \gamma_{t_{i-1}} S_{t_{i-1}}^{2}\left(\sigma \sqrt{\frac{2}{\pi}} \sqrt{n f^{\prime}\left(t_{i-1}\right)} \Delta t_{i}-\mathbf{E}\left|\mathcal{E}_{t_{i-1}}^{t_{i}}\right|\right) \\
R_{1}^{n}(t) & :=\int_{0}^{t}\left(\delta_{u}^{n}-\delta_{u}\right) d S_{u} \\
R_{2}^{n}(t) & :=k_{0} \sum_{i \in J_{1}^{n}(t)}\left(\left|\Delta \delta_{t_{i}}^{n}+\Delta K_{t_{i}}^{n}\right|-\left|\Delta \delta_{t_{i}}^{n}+\Delta L_{t_{i}}^{n}\right|\right) S_{t_{i}} \\
R_{3}^{n}(t) & :=\int_{0}^{t}\left(L_{u}^{n}-K_{u}^{n}\right) d S_{u} .
\end{aligned}
$$

\subsection{Step 2: The mean square residue tends to 0 with rate $n^{\frac{1}{2}+2 p}$.}

The most technical part of this paper is the following. The deviation of the approximating portfolio from the payoff has been written in an integral form by virtue of the Ito formula. The "real world" portfolio may be interpreted as a discretetime approximation of the theoretical portfolio $\widehat{C}\left(t, S_{t}\right)$ yielding the residual terms above. Consequently, the following analysis is mainly based on Taylor approximations involving the successive derivatives of $\widehat{C}$ and so heavily utilizes estimates of the appendix. Standard tools from stochastic calculus are also frequently used. 
Theorem 5.1. The following convergence holds:

$$
n^{\frac{1}{2}+2 p} \mathbf{E}\left(\varepsilon_{t}^{n}\right)^{2} \underset{n \rightarrow \infty}{\longrightarrow} 0 .
$$

To prove this theorem, we show the suitable convergence to 0 concerning the $R_{j}, \quad 0 \leq j \leq 3$.

\section{Lemma 5.2.}

$$
n^{\frac{1}{2}+2 p} \mathbf{E}\left(R_{0}^{n}\right)^{2} \underset{n \rightarrow \infty}{\longrightarrow} 0 .
$$

Proof. We have

$$
\begin{aligned}
\mathbf{E}\left|\mathcal{E}_{t_{i-1}}^{t_{i}}\right|=4 \Phi\left(\frac{\sigma \sqrt{\Delta t_{i}}}{2}\right)-2 & =\sigma \sqrt{\frac{2}{\pi}} \sqrt{\Delta t_{i}}+\left(\Delta t_{i}\right) o(1), \\
\sigma \sqrt{\frac{2}{\pi}} n^{\frac{1}{2}} \sqrt{f^{\prime}\left(t_{i-1}\right)} \Delta t_{i} & =\sigma \sqrt{\frac{2}{\pi}} \sqrt{\Delta t_{i}} \varepsilon_{i}
\end{aligned}
$$

where $\varepsilon_{i}=n^{\frac{1}{2}} \sqrt{\Delta t_{i}} \sqrt{f^{\prime}\left(t_{i-1}\right)}$ verifies

$$
\left|\varepsilon_{i}-1\right| \leq \frac{c \Delta t_{i}}{1-t_{i}}
$$

by virtue of Lemma 6.12 . Hence, there is a constant $C>0$ such that:

$$
\sup _{t}\left|R_{0}^{n}(t)\right| \leq C k_{0} \sum_{i=1}^{n-1} \gamma_{t_{i-1}} S_{t_{i-1}}^{2} \frac{\left(\Delta t_{i}\right)^{\frac{3}{2}}}{1-t_{i}} .
$$

From Corollary 6.5 and Inequalities (4.28-4.32), we deduce the following

$$
\begin{aligned}
n^{\frac{1}{4}+p} \sqrt{\mathbf{E}\left(\sup _{t}\left|R_{0}^{n}(t)\right|\right)^{2}} & \leq C n^{\frac{1}{8}+p} \sum_{i=1}^{n-1} \frac{\left(\Delta t_{i}\right)^{\frac{3}{2}}}{\left(1-t_{i}\right)^{5 / 4}} \\
& \leq C \frac{n^{\frac{1}{8}+p}}{n^{\frac{1}{4}}} \log n \underset{n \rightarrow \infty}{\longrightarrow} 0 .
\end{aligned}
$$

A Taylor formula suggests to write the following splitting:

$$
R_{1}^{n}=\sigma\left(R_{10}^{n}-R_{11}^{n}-R_{12}^{n}-R_{13}^{n}+2 R_{14}^{n}\right),
$$


where

$$
\begin{aligned}
& R_{10}^{n}(t):=\sum_{i \leq n} \gamma_{t_{i-1}} S_{t_{i-1}}^{2} \int_{t_{i-1} \wedge t}^{t_{i} \wedge t} \mathcal{E}_{t_{i-1}}^{u} \frac{S_{u}}{S_{t_{i-1}}} d W_{u} \\
& R_{11}^{n}(t):=\sum_{i=1}^{n-1} \int_{t_{i-1} \wedge t}^{t_{i} \wedge t} \widehat{C}_{x t}\left(t_{i-1}, S_{t_{i-1}}\right)\left(u-t_{i-1}\right) S_{u} d W_{u}, \\
& R_{12}^{n}(t):=\frac{1}{2} \sum_{i=1}^{n-1} S_{t_{i-1}}^{3} \int_{t_{i-1} \wedge t}^{t_{i} \wedge t} \widehat{C}_{x x x}\left(\widetilde{t}_{i-1}, \widetilde{S}_{t_{i-1}}\right)\left(\mathcal{E}_{t_{i-1}}^{u}\right)^{2} \frac{S_{u}}{S_{t_{i-1}}} d W_{u}, \\
& R_{13}^{n}(t):=\frac{1}{2} \sum_{i=1}^{n-1} S_{t_{i-1}} \int_{t_{i-1} \wedge t}^{t_{i} \wedge t} \widehat{C}_{x t t}\left(\widetilde{t}_{i-1}, \widetilde{S}_{t_{i-1}}\right)\left(u-t_{i-1}\right)^{2} \frac{S_{u}}{S_{t_{i-1}}} d W_{u}, \\
& R_{14}^{n}(t):=-\frac{1}{2} \sum_{i=1}^{n-1} S_{t_{i-1}}^{2} \int_{t_{i-1} \wedge t}^{t_{i} \wedge t} \widehat{C}_{x x t}\left(\widetilde{t}_{i-1}, \widetilde{S}_{t_{i-1}}\right) \mathcal{E}_{t_{i-1}}^{u}\left(u-t_{i-1}\right) \frac{S_{u}}{S_{t_{i-1}}} d W_{u} .
\end{aligned}
$$

\section{Lemma 5.3.}

$$
n^{\frac{3}{2}+2 p} \mathbf{E}\left(\sup _{t \in[0,1]} R_{10}^{n}(t)\right)^{2} \underset{n \rightarrow \infty}{\longrightarrow} 0 .
$$

Proof. The Doob inequality yields

$$
n^{\frac{3}{2}+2 p} \mathbf{E} \sup _{t}\left(R_{10}^{n}(t)\right)^{2} \leq 4 n^{\frac{3}{2}+2 p} \mathbf{E}\left(R_{10}^{n}(1)\right)^{2}
$$

where the r.h.s tends to 0 as shown below. Indeed, by the independence of the increments of the Wiener process, we write:

$$
\mathbf{E}\left(R_{10}^{n}(1)\right)^{2}=\sigma^{2} \sum_{i=1}^{n} \Lambda_{t_{i-1}} \int_{t_{i-1}}^{t_{i}} \mathbf{E}\left(\mathcal{E}_{t_{i-1}}^{u}\right)^{2} \frac{S_{u}^{2}}{S_{t_{i-1}}^{2}} d u
$$

where

$$
\Lambda_{t}:=\mathbf{E} \widehat{C}_{x x}^{2}\left(t, S_{t}\right) S_{t}^{4} .
$$

It is easy to check the following asymptotic

$$
\mathbf{E}\left(\mathcal{E}_{t_{i-1}}^{u}\right)^{2} \frac{S_{u}^{2}}{S_{t_{i-1}}^{2}}=\sigma^{2}\left(u-t_{i-1}\right)+\left(u-t_{i-1}\right) O\left(n^{-1}\right) .
$$

Therefore

$$
\mathbf{E}\left(R_{10}^{n}(1)\right)^{2}=\frac{\sigma^{4}}{2} \sum_{i \leq n} \Lambda_{t_{i-1}}\left(\Delta t_{i}\right)^{2}\left(1+O\left(n^{-1}\right)\right)
$$

where $\Delta t_{i}=g^{\prime}\left(\theta_{i}\right) / n$ with $\theta_{i} \in[(i-1) / n, i / n]$. We then deduce

$$
n^{\frac{3}{2}+2 p} \mathbf{E}\left(R_{10}^{n}(1)\right)^{2}=\frac{\sigma^{4}\left(1+O\left(n^{-1}\right)\right)}{2} \sum_{i \leq n} \Lambda_{t_{i-1}}\left(\Delta t_{i} n\right) \frac{\Delta t_{i} n^{\frac{1}{2}+2 p}}{x_{i-1}-x_{i}}\left(x_{i-1}-x_{i}\right)
$$


where $x_{i}=\rho_{t_{i}}^{2}$. So, we have:

$$
n^{\frac{3}{2}+2 p} \mathbf{E}\left(R_{10}^{n}(1)\right)^{2}=\frac{\sigma^{4}\left(1+O\left(n^{-1}\right)\right)}{2} \int_{0}^{\rho_{0}^{2}} f_{n}(x) d x
$$

where

$$
f_{n}(x)=\sum_{i=1}^{n} \Lambda_{t_{i-1}}\left(\Delta t_{i} n\right) \frac{\Delta t_{i} n^{\frac{1}{2}+2 p}}{x_{i-1}-x_{i}} 1_{\left(x_{i}, x_{i-1}\right]}(x) .
$$

Let us remark the abuse of notations $\rho_{0}^{2}=\rho_{0, n}^{2}$ and $t_{i}=t\left(n, x_{i}\right)$ as previously mentioned.

First, let us show that $f_{n}$ satisfies the dominated convergence bound condition. If $x \in\left(x_{i}, x_{i-1}\right]$ then from Corollary 6.5 , we have

$$
0 \leq \Lambda_{t_{i-1}} \leq \frac{C}{\sqrt{x_{i-1}}} e^{-x_{i-1} / 4} \leq \frac{C}{\sqrt{x}} e^{-x / 4} .
$$

Thus, from (4.33) we obtain

$$
f_{n}(x) \leq \frac{C}{\sqrt{x}} e^{-x / 4}(1+x) .
$$

Regarding the pointwise convergence of $f_{n}$, for a given $x \in\left(x_{i}, x_{i-1}\right]$, there exists $u \in\left[t_{i-1}, t_{i}\right)$ such that $x=\rho_{u}^{2} \geq c n^{\frac{1}{2}}(1-u)$. It follows that not only $u \rightarrow 1$ but also $t_{i}, t_{i-1} \rightarrow 1$. Recall that $\Delta t_{i}=g^{\prime}\left(\theta_{i}\right) n^{-1}$ where $\theta_{i} \in[(i-1) / n, i / n]$. Thus $g\left(\theta_{i}\right) \rightarrow 1$ and $\theta_{i} \rightarrow 1$ since $f$ is continuous. Therefore $\Delta t_{i} n \rightarrow g^{\prime}(1)=0$. Moreover, note that

$$
\Lambda_{t_{i-1}}=\frac{1}{x_{i-1}} \int_{-\infty}^{\infty} e^{2 \sigma \sqrt{t_{i-1}} z-\sigma^{2} t_{i-1}} \Upsilon_{i}(z) \varphi(z) d z
$$

where

$$
\Upsilon_{i}(z)=\left(\int_{-\infty}^{\infty} h^{\prime}\left(e^{\sigma \sqrt{t_{i-1}} z-\frac{\sigma^{2} t_{i-1}}{2}+\sqrt{x_{i-1}} y+\frac{x_{i-1}}{2}}\right) y \varphi(y) d y\right)^{2} .
$$

Applying the Lebesgue theorem, we deduce that $\Lambda_{t_{i-1}}$ converges to

$$
\Lambda(x):=\frac{1}{x} \int_{-\infty}^{\infty} e^{2 \sigma z-\sigma^{2}}\left(\int_{-\infty}^{\infty} h^{\prime}\left(e^{\sigma z-\frac{\sigma^{2}}{2}+\sqrt{x} y+\frac{x}{2}}\right) y \varphi(y) d y\right)^{2} \varphi(z) d z .
$$

Finally, together with (4.34), $f_{n} \underset{n \rightarrow \infty}{\longrightarrow} 0$ a.e. We then apply the Lebesgue theorem to conclude the following limit

$$
\frac{\sigma^{4}\left(1+O\left(n^{-1}\right)\right)}{2} \int_{0}^{\rho_{0}^{2}} f_{n}(x) d x \underset{n \rightarrow \infty}{\longrightarrow} 0
$$

Lemma 5.4. $n^{\frac{1}{2}+2 p} \mathbf{E}\left(\sup _{t} R_{11}^{n}(t)\right)^{2} \underset{n \rightarrow \infty}{\longrightarrow} 0$. 
Proof. Using the Doob inequality, we obtain that $\mathbf{E}\left(\sup _{t} R_{11}^{n}(t)\right)^{2} \leq 4 \mathbf{E}\left(R_{11}^{n}(1)\right)^{2}$. By independence of the increments of the Wiener process, we deduce that $n^{\frac{1}{2}+2 p} \mathbf{E}\left(R_{11}^{n}(1)\right)^{2}=n^{\frac{1}{2}+2 p} \sum_{i=1}^{n-1} \mathbf{E} \widehat{C}_{x t}^{2}\left(t_{i-1}, S_{t_{i-1}}\right) S_{t_{i-1}}^{2} \int_{t_{i-1}}^{t_{i}}\left(u-t_{i-1}\right)^{2} \mathbf{E}\left(\frac{S_{u}}{S_{t_{i-1}}}\right)^{2} d u$.

It follows that

$$
n^{\frac{1}{2}+2 p} \mathbf{E}\left(R_{11}^{n}(1)\right)^{2} \leq c n^{\frac{1}{2}+2 p} \sum_{i=1}^{n-1} \mathbf{E} \widehat{C}_{x t}^{2}\left(t_{i-1}, S_{t_{i-1}}\right) S_{t_{i-1}}^{2}\left(\Delta t_{i}\right)^{3} \leq c n^{-\frac{1}{4}+2 p} \log n,
$$

since Corollary 6.14 gives

$$
\mathbf{E} \widehat{C}_{x t}^{2}\left(t_{i-1}, S_{t_{i-1}}\right) S_{t_{i-1}}^{2} \leq c \frac{n^{\frac{1}{4}} f^{\prime}\left(t_{i-1}\right)}{\left(1-t_{i-1}\right)^{\frac{3}{2}}}
$$

where $n f^{\prime}\left(t_{i-1}\right) \Delta t_{i}$ is bounded. We then conclude.

Lemma 5.5. $n^{\frac{1}{2}+2 p} \mathbf{E}\left(\sup _{t} R_{12}^{n}(t)\right)^{2} \underset{n \rightarrow \infty}{\longrightarrow} 0$.

Proof. As previously, we have the Doob inequality $\mathbf{E}\left(\sup _{t} R_{12}^{n}(t)\right)^{2} \leq 4 \mathbf{E}\left(R_{12}^{n}(1)\right)^{2}$ and the equality

$$
4 \mathbf{E}\left(R_{12}^{n}(1)\right)^{2}=\sum_{i=1}^{n-1} \int_{t_{i-1}}^{t_{i}} \mathbf{E}\left(\widehat{C}_{x x x}^{2}\left(\widetilde{t}_{i-1}, \widetilde{S}_{t_{i-1}}\right) S_{t_{i-1}}^{6}\left(1-\frac{S_{t}}{S_{t_{i-1}}}\right)^{4} \frac{S_{t}^{2}}{S_{t_{i-1}}^{2}}\right) d t .
$$

From (6.125), there exists a constant $C$ such that:

$$
\mathbf{E} \widehat{C}_{x x x}^{4}\left(\widetilde{t}_{i-1}, \widetilde{S}_{t_{i-1}}\right) \leq \frac{C}{\rho_{t_{i}}^{8}} .
$$

Using the Cauchy-Schwarz inequality and (4.23) with $m=8$, we deduce that

$$
\begin{aligned}
n^{\frac{1}{2}+2 p} \mathbf{E}\left(R_{12}^{n}(1)\right)^{2} & \leq C n^{\frac{1}{2}+2 p} \sum_{i=1}^{n-1} \frac{\left(\Delta t_{i}\right)^{3}}{n\left(1-t_{i}\right)^{2}} \\
& \leq C \frac{n^{2 p} \log n}{n^{\frac{3}{2}}}
\end{aligned}
$$

which proves the desired convergence to 0 .

Lemma 5.6. $n^{\frac{1}{2}+2 p} \mathbf{E}\left(\sup _{t} R_{13}^{n}(t)\right)^{2} \underset{n \rightarrow \infty}{\longrightarrow} 0$.

Proof. We still consider the Doob inequality $\mathbf{E}\left(\sup _{t} R_{13}^{n}(t)\right)^{2} \leq 4 \mathbf{E}\left(R_{13}^{n}(1)\right)^{2}$ and

$$
4 \mathbf{E}\left(R_{13}^{n}(1)\right)^{2} \leq \sum_{i=1}^{n-1} \int_{t_{i-1}}^{t_{i}} \mathbf{E}\left(\widehat{C}_{x t t}^{2}\left(\widetilde{t}_{i-1}, \widetilde{S}_{t_{i-1}}\right)\left(t-t_{i-1}\right)^{4} S_{t}^{2}\right) d t .
$$

Moreover, using Lemma 6.19 and the Cauchy-Schwarz inequality, we deduce that

$$
\mathbf{E}\left(\widehat{C}_{x t t}^{2}\left(\widetilde{t}_{i-1}, \widetilde{S}_{t_{i-1}}\right) S_{t}^{2}\right) \leq \frac{c}{\left(1-t_{i}\right)^{4}} .
$$


Then, we obtain

$$
n^{\frac{1}{2}+2 p} \mathbf{E}\left(R_{13}^{n}(1)\right)^{2} \leq C n^{\frac{1}{2}+2 p} \sum_{i=1}^{n-1} \frac{\left(\Delta t_{i}\right)^{5}}{\left(1-t_{i}\right)^{4}} \leq C n^{-\frac{1}{2}+2 p} \log n .
$$

The conclusion follows.

Lemma 5.7. $n^{\frac{1}{2}+2 p} \mathbf{E}\left(\sup _{t} R_{14}^{n}(t)\right)^{2} \underset{n \rightarrow \infty}{\longrightarrow} 0$.

Proof. We use the Doob inequality $\mathbf{E}\left(\sup _{t} R_{14}^{n}(t)\right)^{2} \leq 4 \mathbf{E}\left(R_{14}^{n}(1)\right)^{2}$ and the equality

$4 \mathbf{E}\left(R_{14}^{n}(1)\right)^{2}=\sum_{i=1}^{n-1} \int_{t_{i-1}}^{t_{i}} \mathbf{E}\left(S_{t_{i-1}}^{4} \widehat{C}_{x x t}^{2}\left(\widetilde{t}_{i-1}, \widetilde{S}_{t_{i-1}}\right)\left(1-\frac{S_{t}}{S_{t_{i-1}}}\right)^{2}\left(t-t_{i-1}\right)^{2} \frac{S_{t}^{2}}{S_{t_{i-1}}^{2}}\right) d t$.

From (6.126), we deduce that

$$
\mathbf{E}\left(S_{t_{i-1}}^{4} \widehat{C}_{x x t}^{2}\left(\widetilde{t}_{i-1}, \widetilde{S}_{t_{i-1}}\right)\left(1-\frac{S_{t}}{S_{t_{i-1}}}\right)^{2} \frac{S_{t}^{2}}{S_{t_{i-1}}^{2}}\right) \leq c \frac{t-t_{i-1}}{\left(1-t_{i}\right)^{3}} .
$$

Then,

$$
n^{\frac{1}{2}+2 p} \mathbf{E}\left(R_{14}^{n}(1)\right)^{2} \leq c n^{\frac{1}{2}+2 p} \sum_{i=1}^{n-1} \frac{\left(t_{i}-t_{i-1}\right)^{4}}{\left(1-t_{i}\right)^{3}} \leq c n^{-\frac{1}{2}+2 p} \log n .
$$

Thus, we can conclude.

Let us now study the residual term $R_{2}^{n}$. Again, a Taylor formula suggests to write the following splitting:

$$
R_{2}^{n}=R_{20}^{n}+\cdots+R_{24}^{n}
$$

where

$$
\begin{aligned}
& R_{20}^{n}(t):=\sigma k_{0} \sqrt{\frac{2}{\pi}} n^{\frac{1}{2}} \int_{t_{n}^{-}(t)}^{t} S_{u}^{2} \gamma_{u} \sqrt{f^{\prime}(u)} d u \\
& R_{21}^{n}(t):=\sigma k_{0} n^{\frac{1}{2}} \sqrt{\frac{2}{\pi}} \sum_{i \in J_{1}^{n}(t)} \int_{t_{i-1}}^{t_{i}}\left(S_{u}^{2} \gamma_{u} \sqrt{f^{\prime}(u)}-S_{t_{i-1}}^{2} \gamma_{t_{i-1}} \sqrt{f^{\prime}\left(t_{i-1}\right)}\right) d u \\
& R_{22}^{n}(t):=k_{n} \sum_{i \in J_{1}^{n}(t)} \gamma_{t_{i-1}}\left|S_{t_{i}}-S_{t_{i-1}}\right|\left(S_{t_{i-1}}-S_{t_{i}}\right) \\
& R_{23}^{n}(t):=k_{0} \sum_{i \in J_{1}^{n}(t)} \Theta_{i}\left(S_{t_{i}}-S_{t_{i-1}}\right) \\
& R_{24}^{n}(t):=k_{0} \sum_{i \in J_{1}^{n}(t)} \Theta_{i} S_{t_{i-1}}, \\
& \Theta_{i}:=\gamma_{t_{i-1}}\left|S_{t_{i}}-S_{t_{i-1}}\right|-\left|\widehat{C}_{x}\left(t_{i}, S_{t_{i}}\right)-\widehat{C}_{x}\left(t_{i-1}, S_{t_{i-1}}\right)+\Delta K_{t_{i}}^{n}\right| .
\end{aligned}
$$


Lemma 5.8. $n^{\frac{1}{2}+2 p} \mathbf{E}\left(R_{20}^{n}(1)\right)^{2} \underset{n \rightarrow \infty}{\longrightarrow} 0$.

Proof. We have

$$
n^{\frac{1}{2}+2 p} \mathbf{E}\left(R_{20}^{n}(1)\right)^{2}=c n^{\frac{3}{2}+2 p} \mathbf{E} \int_{\left[t_{n-1}, 1\right]^{2}} S_{u}^{2} \gamma_{u} S_{v}^{2} \gamma_{v} \sqrt{f^{\prime}(u)} \sqrt{f^{\prime}(v)} d u d v
$$

We use the Cauchy-Schwarz inequality, Inequalities (6.5) and (4.30). From the explicit formula of $f^{\prime}$, we thus obtain

$$
\begin{aligned}
n^{\frac{1}{2}+2 p} \mathbf{E}\left(R_{20}^{n}(1)\right)^{2} & \leq c n^{1+2 p} \int_{\left[t_{n-1}, 1\right]^{2}} \frac{d u d v}{(1-u)^{5 / 8-3 /(8 \mu)}(1-v)^{5 / 8-3 /(8 \mu)}}, \\
& \leq c \frac{n^{1+2 p}}{n^{3 / 4+3 /(4 \mu)}} .
\end{aligned}
$$

Since $\mu \in(1,2]$,

$$
\frac{3}{4}+\frac{3}{4 \mu}-(1+2 p)=\frac{-3 \mu^{2}+5 \mu+3}{4 \mu(\mu+1)}>0
$$

so that we can conclude.

Lemma 5.9. $n^{\frac{1}{2}+2 p} \mathbf{E}\left(\sup _{t} R_{21}^{n}(t)\right)^{2} \underset{n \rightarrow \infty}{\longrightarrow} 0$.

Proof. Let us consider $\Psi(t, x):=x^{2} \widehat{C}_{x x}(t, x) \sqrt{f^{\prime}(t)}$. The Ito formula yields

$$
\begin{aligned}
\Psi\left(t, S_{t}\right)=\quad & \Psi\left(t_{i-1}, S_{t_{i-1}}\right)+\int_{t_{i-1}}^{t} \frac{\partial \Psi}{\partial x}\left(u, S_{u}\right) \sigma S_{u} d W_{u}+\int_{t_{i-1}}^{t} \frac{\partial \Psi}{\partial t}\left(u, S_{u}\right) d u \\
& +\frac{1}{2} \int_{t_{i-1}}^{t} \frac{\partial^{2} \Psi}{\partial x^{2}}\left(u, S_{u}\right) \sigma^{2} S_{u}^{2} d u,
\end{aligned}
$$

where

$$
\begin{aligned}
\frac{\partial \Psi}{\partial t}(t, x) & =x^{2}\left[\widehat{C}_{x x t}(t, x) \sqrt{f^{\prime}(t)}+\widehat{C}_{x x}(t, x) \frac{f^{\prime \prime}(t)}{2 \sqrt{f^{\prime}(t)}}\right], \\
\frac{\partial \Psi}{\partial x}(t, x) & =\left[2 x \widehat{C}_{x x}(t, x)+x^{2} \widehat{C}_{x x x}(t, x)\right] \sqrt{f^{\prime}(t)}, \\
\frac{\partial^{2} \Psi}{\partial x^{2}}(t, x) & =\left[2 \widehat{C}_{x x}(t, x)+4 x \widehat{C}_{x x x}(t, x)+x^{2} \widehat{C}_{x x x x}(t, x)\right] \sqrt{f^{\prime}(t)} .
\end{aligned}
$$

If we set $X_{t}=S_{t}^{2} \widehat{C}_{x x}(t, x) \sqrt{f^{\prime}(t)}$ then $d X_{t}=\mu_{t} d t+\beta_{t} d W_{t}$ where

$$
\begin{aligned}
\mu_{t} & =\frac{\partial \Psi}{\partial t}\left(t, S_{t}\right)+\frac{1}{2} \frac{\partial^{2} \Psi}{\partial x^{2}}\left(t, S_{t}\right) \sigma^{2} S_{t}^{2} \\
\beta_{t} & =\frac{\partial \Psi}{\partial x}\left(t, S_{t}\right) \sigma S_{t} .
\end{aligned}
$$

We write $n^{\frac{1}{4}+p} R_{21}^{n}(t)=A_{t}^{n}+B_{t}^{n}$ with

$$
\begin{aligned}
A_{t}^{n} & :=\sigma k_{0} n^{\frac{3}{4}+p} \sqrt{\frac{2}{\pi}} \sum_{i \in J_{1}^{n}(t)} \int_{t_{i-1}}^{t_{i}}\left(\int_{t_{i-1}}^{t} \beta_{u} d W_{u}\right) d t, \\
B_{t}^{n} & :=\sigma k_{0} n^{\frac{3}{4}+p} \sqrt{\frac{2}{\pi}} \sum_{i \in J_{1}^{n}(t)} \int_{t_{i-1}}^{t_{i}}\left(\int_{t_{i-1}}^{t} \mu_{u} d u\right) d t .
\end{aligned}
$$


From (6.120), there exists a constant $C$ such that:

$$
\mathbf{E} \beta_{t}^{2} \leq c\left(\mathbf{E} S_{t}^{4} \delta_{t}^{2}+\mathbf{E} S_{t}^{6} \widehat{C}_{x x x}^{2}\left(t, S_{t}\right)\right) f^{\prime}(t) \leq \frac{C f^{\prime}(t)^{\frac{1}{4}}}{n^{\frac{3}{4}}(1-t)^{\frac{3}{2}}} .
$$

Using A1, we claim that there exists a constant $\widetilde{c}$ such that

$$
\frac{\left|f^{\prime \prime}(t)\right|}{\sqrt{f^{\prime}(t)}}=\frac{\widetilde{c}}{(1-t)^{\frac{3}{2}-1 /(2 / \mu)}} .
$$

Thus, using (6.119-6.124), we obtain some constant $C$ such that the following inequality holds:

$$
\mathbf{E} \mu_{t}^{2} \leq \frac{c(1-t)^{3 /(4 \mu)}}{n^{\frac{1}{4}}(1-t)^{13 / 4}}+\frac{c(1-t)^{3 /(4 \mu)}}{n^{3 / 4}(1-t)^{7 / 4}}+\frac{c}{n^{5 / 4}(1-t)^{9 / 4+1 /(4 \mu)}}
$$

By means of the stochastic Fubini theorem, we obtain that

$$
A_{t}^{n}=\sigma k_{0} n^{\frac{3}{4}+2 p} \sqrt{\frac{2}{\pi}} \sum_{i \in J_{1}^{n}(t)} \int_{t_{i-1}}^{t_{i}}\left(t_{i}-u\right) \beta_{u} d W_{u} .
$$

Since the Doob inequality $\mathbf{E}\left(\sup _{t} A_{t}^{n}\right)^{2} \leq 4 \mathbf{E}\left(A_{1}^{n}\right)^{2}$ holds, it suffices to estimate $\mathbf{E}\left(A_{1}^{n}\right)^{2}$. From the boundedness of $\sqrt{\left(t_{i}-u\right) /(1-u)}$ and $f^{\prime}(u)\left(t_{i}-u\right) n$ on $u \in$ $\left[t_{i-1}, t_{i}\right)$, we deduce the following estimates:

$$
\begin{aligned}
\mathbf{E}\left(A_{1}^{n}\right)^{2} & \leq c n^{\frac{3}{2}+2 p} \sum_{i=1}^{n-1} \int_{t_{i-1}}^{t_{i}}\left(t_{i}-u\right)^{2} \mathbf{E} \beta_{u}^{2} d u \\
& \leq c n^{\frac{3}{2}+2 p} \sum_{i=1}^{n-1} \int_{t_{i-1}}^{t_{i}} \frac{\left(t_{i}-u\right)^{2} f^{\prime}(u)^{\frac{1}{4}}}{n^{3 / 4}(1-u)^{\frac{3}{2}}} d u \\
& \leq \frac{c n^{2 p}}{n^{\frac{1}{4}}} \sum_{i=1}^{n-1} \int_{t_{i-1}}^{t_{i}} \frac{\left(t_{i}-u\right)}{(1-u)^{\frac{3}{2}}} d u \leq c \frac{n^{2 p} \log n}{n^{3 / 4}} \underset{n \rightarrow \infty}{\longrightarrow} 0 .
\end{aligned}
$$

Then, we conclude that $\mathbf{E}\left(\sup _{t} A_{t}^{n}\right)^{2} \underset{n \rightarrow \infty}{\longrightarrow} 0$.

Secondly, we write:

$$
\begin{aligned}
B_{t}^{n} & =c n^{3 / 4+p} \sum_{i \in J_{1}^{n}(t)} \int_{t_{i-1}}^{t_{i}} \mu_{u} \int_{t_{i-1}}^{t_{i}} \mathbf{1}_{t \geq u} d t d u \\
& =c n^{3 / 4+p} \sum_{i \in J_{1}^{n}(t)} \int_{t_{i-1}}^{t_{i}}\left(t_{i}-u\right) \mu_{u} d u .
\end{aligned}
$$

Then,

$$
\sup _{t}\left|B_{t}^{n}\right| \leq c n^{3 / 4+p} \sum_{i=1}^{n-1} \int_{t_{i-1}}^{t_{i}}\left(t_{i}-u\right)\left|\mu_{u}\right| d u .
$$


Thus there exists a constant $c$ such that $\mathbf{E} \sup _{t}\left|B_{t}^{n}\right|^{2} \leq c n^{\frac{3}{2}+2 p} \Upsilon^{n}$ where

$$
\begin{aligned}
\Upsilon^{n} & =\mathbf{E}\left(\int_{0}^{1} \sum_{i=1}^{n-1}\left(t_{i}-u\right)\left|\mu_{u}\right| \mathbf{1}_{\left(t_{i-1}, t_{i}\right]}(u) d u\right)^{2}, \\
& =\mathbf{E} \int_{0}^{1} \int_{0}^{1} \sum_{i, j=1}^{n-1}\left(t_{i}-u\right)\left(t_{j}-v\right)\left|\mu_{u}\right|\left|\mu_{v}\right| \mathbf{1}_{\left(t_{i-1}, t_{i}\right]}(u) \mathbf{1}_{\left(t_{j-1}, t_{j}\right]}(v) d u d v .
\end{aligned}
$$

Using the Cauchy-Schwarz inequality and (5.55), we can then bound $\Upsilon^{n}$ :

$$
\begin{aligned}
\Upsilon^{n} & \leq \int_{0}^{1} \int_{0}^{1} \sum_{i, j=1}^{n-1}\left(t_{i}-u\right)\left(t_{j}-v\right)\left(\mathbf{E} \mu_{u}^{2}\right)^{\frac{1}{2}}\left(\mathbf{E} \mu_{v}^{2}\right)^{\frac{1}{2}} \mathbf{1}_{\left(t_{i-1}, t_{i}\right]}(u) \mathbf{1}_{\left(t_{j-1}, t_{j}\right]}(v) d u d v \\
& \leq\left(\int_{0}^{1} \sum_{i=1}^{n-1}\left(t_{i}-u\right)\left(\mathbf{E} \mu_{u}^{2}\right)^{\frac{1}{2}} \mathbf{1}_{\left(t_{i-1}, t_{i}\right]}(u) d u\right)^{2}, \\
& \leq c\left(\Upsilon^{1 n}+\Upsilon^{2 n}+\Upsilon^{3 n}\right)
\end{aligned}
$$

where

$$
\Upsilon^{1 n} \leq\left(\sum_{i \leq n-1} \frac{\left(\Delta t_{i}\right)^{2}}{\left(1-t_{i}\right)} \frac{1}{n^{1 / 8}(1-t)^{5 / 8-3 /(8 \mu)}}\right)^{2} \leq \frac{c \log n}{n^{1+3 /(4 \mu)}}
$$

In a same way, we obtain the following inequalities

$$
\begin{aligned}
\Upsilon^{2 n} & \leq\left(\sum_{i \leq n-1} \frac{\left(\Delta t_{i}\right)^{2}}{n^{3 / 8}\left(1-t_{i}\right)^{7 / 8}}\right)^{2} \leq \frac{C}{n^{\frac{5}{4}}} \\
\Upsilon^{3 n} & \leq\left(\sum_{i \leq n-1} \frac{\left(\Delta t_{i}\right)^{2}}{n^{5 / 8}\left(1-t_{i}\right)^{1+(1 / 8+1 /(8 \mu))}}\right)^{2} \leq \frac{C t \log n}{n^{7 / 2+1 /(4 \mu)}} .
\end{aligned}
$$

Then, from inequalities (5.56), (5.57) and (5.58) we deduce that

$$
\mathbf{E} \sup _{t}\left|B_{t}^{n}\right|^{2} \leq \frac{C n^{\frac{3}{2}+2 p} \log n}{n^{1+3 /(4 \mu)}} \leq \frac{C \log n}{n^{3 /(4 \mu)-\frac{1}{2}-2 p}}
$$

where

$$
3 /(4 \mu)-\frac{1}{2}-2 p=\frac{-4 \mu^{2}+3 \mu+3}{4 \mu(\mu+1)} .
$$

Assumption $(A 1)$ yields $-4 \mu^{2}+3 \mu+3>0$. Hence the result follows.

Lemma 5.10. $n^{\frac{1}{2}+2 p} \mathbf{E}\left(\sup _{t} R_{22}^{n}(t)\right)^{2} \underset{n \rightarrow \infty}{\longrightarrow} 0$.

Proof. We write

$$
-R_{22}^{n}(t)=k_{n} \sum_{i \in J_{1}^{n}(t)} \gamma_{t_{i-1}} S_{t_{i-1}}^{2}\left\{\mathcal{E}_{t_{i-1}}^{t_{i}}\right\}_{s}^{2}=U^{n}(t)+V^{n}(t)
$$


where $U^{n}$ is a martingale defined as

$$
U^{n}(t):=k_{0} \sum_{i \in J_{1}^{n}(t)} \gamma_{t_{i-1}} S_{t_{i-1}}^{2}\left(\left\{\mathcal{E}_{t_{i-1}}^{t_{i}}\right\}_{s}^{2}-\mathbf{E}\left\{\mathcal{E}_{t_{i-1}}^{t_{i}}\right\}_{s}^{2}\right)
$$

and

$$
V^{n}(t):=k_{0} \sum_{i \in J_{1}^{n}(t)} \gamma_{t_{i-1}} S_{t_{i-1}}^{2} \mathbf{E}\left\{\mathcal{E}_{t_{i-1}}^{t_{i}}\right\}_{s}^{2} .
$$

Recall that from Lemma 4.1

$$
\mathbf{E}\left\{\mathcal{E}_{t_{i-1}}^{t_{i}}\right\}_{s}^{2}=k\left(\Delta t_{j}\right)^{\frac{3}{2}}\left(1+o\left(n^{-\frac{1}{4}}\right)\right) .
$$

We deduce that for $n$ large enough, $0 \leq \mathbf{E}\left\{\mathcal{E}_{t_{i-1}}^{t_{i}}\right\}_{s}^{2} \leq c\left(\Delta t_{i}\right)^{\frac{3}{2}}$. Using the Doob inequality $\mathbf{E}\left(\sup _{t} U^{n}(t)\right)^{2} \leq 4 \mathbf{E}\left(U^{n}(1)\right)^{2}$, it suffices to estimate $\mathbf{E}\left(U^{n}(1)\right)^{2}$. The independence of the increments of the Brownian motion implies the equality

$$
\mathbf{E}\left(U^{n}(1)\right)^{2}=k_{0}^{2} \sum_{i=1}^{n-1} \mathbf{E} \widehat{C}_{x x}^{2}\left(t_{i-1}, S_{t_{i-1}}\right) S_{t_{i-1}}^{4} \mathbf{E}\left(\left\{\mathcal{E}_{t_{i-1}}^{t_{i}}\right\}_{s}^{2}-\mathbf{E}\left\{\mathcal{E}_{t_{i-1}}^{t_{i}}\right\}_{s}^{2}\right)^{2} .
$$

Then, there exists a constant $C$ such that

$$
n^{\frac{1}{2}+2 p} \mathbf{E}\left(U^{n}(1)\right)^{2} \leq \frac{C n^{2 p}}{n^{\frac{1}{4}}} \underset{n \rightarrow \infty}{\longrightarrow} 0 .
$$

At last, for $n$ large enough, $\mathbf{E}\left\{\mathcal{E}_{t_{i-1}}^{t_{i}}\right\}_{s}^{2} \geq 0$. Hence, $0 \leq \sup _{t} V^{n}(t) \leq N^{n}(1)$. In order to prove that $n^{\frac{1}{2}+2 p} \mathbf{E} V^{n}(1)^{2} \underset{n \rightarrow \infty}{\longrightarrow} 0$, we first analyze the following sum

$$
n^{\frac{1}{2}+2 p} k_{0}^{2} \sum_{i=1}^{n-1} \mathbf{E} \widehat{C}_{x x}^{2}\left(t_{i-1}, S_{t_{i-1}}\right) S_{t_{i-1}}^{4}\left(\mathbf{E}\left\{\mathcal{E}_{t_{i-1}}^{t_{i}}\right\}_{s}^{2}\right)^{2} \leq \frac{c n^{2 p}}{n^{7 / 4}} \underset{n \rightarrow \infty}{\longrightarrow} 0 .
$$

Using the Cauchy-Schwarz inequality, we also have

$$
n^{\frac{1}{2}+2 p} \sum_{t_{i}<t_{j} \leq t_{n-1}} \mathbf{E} \gamma_{t_{i-1}} S_{t_{i-1}}^{2} \gamma_{t_{j-1}} S_{t_{j-1}}^{2} \mathbf{E}\left\{\mathcal{E}_{t_{i-1}}^{t_{i}}\right\}_{s}^{2} \mathbf{E}\left\{\mathcal{E}_{t_{j-1}}^{t_{j}}\right\}_{s}^{2} \leq \frac{c n^{2 p}}{n} \underset{n \rightarrow \infty}{\longrightarrow} 0 .
$$

We then deduce that $n^{\frac{1}{2}+2 p} \mathbf{E} V^{n}(1)^{2} \underset{n \rightarrow \infty}{\longrightarrow} 0$ and finally

$$
n^{\frac{1}{2}+2 p} \mathbf{E}\left(\sup _{t} R_{22}^{n}(t)\right)^{2} \underset{n \rightarrow \infty}{\longrightarrow} 0 .
$$

Lemma 5.11. $n^{\frac{1}{2}+2 p} \mathbf{E}\left(\sup _{t} R_{23}^{n}(t)\right)^{2} \underset{n \rightarrow \infty}{\longrightarrow} 0$.

Proof. We write $R_{23}^{n}(t)=R_{231}^{n}(t)+R_{232}^{n}(t)$ where

$$
\begin{aligned}
& R_{231}^{n}(t):=k_{0} \sum_{i \in J_{1}^{n}(t)} \Theta_{i}^{1}\left(S_{t_{i}}-S_{t_{i-1}}\right) \\
& R_{232}^{n}(t):=k_{0} \sum_{i \in J_{1}^{n}(t)}\left(\Theta_{i}-\Theta_{i}^{1}\right)\left(S_{t_{i}}-S_{t_{i-1}}\right)
\end{aligned}
$$


with

$$
\Theta_{i}^{1}:=\gamma_{t_{i-1}}\left|S_{t_{i}}-S_{t_{i-1}}\right|-\left|\widehat{C}_{x}\left(t_{i}, S_{t_{i}}\right)-\widehat{C}_{x}\left(t_{i-1}, S_{t_{i-1}}\right)\right|
$$

We note that $\sup _{t}\left|R_{231}^{n}(t)\right|$ is bounded by

$$
k_{0} \sum_{i=1}^{n-1}\left|\widehat{C}_{x}\left(t_{i}, S_{t_{i}}\right)-\widehat{C}_{x}\left(t_{i-1}, S_{t_{i-1}}\right)-\gamma_{t_{i-1}}\left(S_{t_{i}}-S_{t_{i-1}}\right)\right|\left|S_{t_{i}}-S_{t_{i-1}}\right| .
$$

Applying Taylor's formula to the difference $\widehat{C}_{x}\left(t_{i}, S_{t_{i}}\right)-\widehat{C}_{x}\left(t_{i-1}, S_{t_{i-1}}\right)$ it is sufficient to estimate the following sums $(5.59), \cdots,(5.62)$. The first one satisfies

$$
n^{\frac{1}{4}+p}\left\|k_{0} \sum_{i=1}^{n-1} \widehat{C}_{x t}\left(t_{i-1}, S_{t_{i-1}}\right)\left(\Delta t_{i}\right)\left(S_{t_{i}}-S_{t_{i-1}}\right)\right\|_{2} \leq C \frac{n^{p}}{n^{1 / 8}} \rightarrow 0
$$

Indeed, from Corollary 6.14, we deduce that:

$$
\mathbf{E} \widehat{C}_{x t}^{2}\left(t_{i-1}, S_{t_{i-1}}\right)\left(\Delta t_{i}\right)^{2}\left(S_{t_{i}}-S_{t_{i-1}}\right)^{2} \leq C \frac{\left(\Delta t_{i}\right)^{3} n^{\frac{1}{4}} f^{\prime}\left(t_{i-1}\right)^{\frac{1}{4}}}{\left(1-t_{i}\right)^{\frac{3}{2}}} .
$$

The second one verifies

$$
(5.60) n^{\frac{1}{4}+p}\left\|k_{0} \sum_{i=1}^{n-1} \widehat{C}_{x x x}\left(\widetilde{t}_{i-1}, \widetilde{S}_{-} t_{i-1}\right)\left(S_{t_{i}}-S_{t_{i-1}}\right)^{3}\right\|_{2} \leq C \frac{n^{p} \log n}{n^{\frac{1}{2}}} \rightarrow 0 .
$$

Thirdly, from (6.126), we deduce that

$$
\mathbf{E} \widehat{C}_{x x t}^{2}\left(\widetilde{t}_{i-1}, \widetilde{S}_{t_{i-1}}\right)\left(S_{t_{i}}-S_{t_{i-1}}\right)^{4}\left(\Delta t_{i}\right)^{2} \leq \frac{C\left(\Delta t_{i}\right)^{4}}{\left(1-t_{i}\right)^{3}}
$$

and it follows that

$$
(5.61) n^{\frac{1}{4}+p}\left\|k_{0} \sum_{i=1}^{n-1} \widehat{C}_{x x t}\left(\widetilde{t}_{i-1}, \widetilde{S}_{t_{i-1}}\right)\left(S_{t_{i}}-S_{t_{i-1}}\right)^{2} \Delta t_{i}\right\|_{2} \leq C \frac{n^{p} \log n}{n^{\frac{1}{4}}} \underset{n \rightarrow \infty}{\longrightarrow} 0 .
$$

Finally, from Lemma 6.19, we get that

$$
\mathbf{E} \widehat{C}_{x t t}^{2}\left(\widetilde{t}_{i-1}, \widetilde{S}_{t_{i-1}}\right)\left(S_{t_{i}}-S_{t_{i-1}}\right)^{2}\left(\Delta t_{i}\right)^{4} \leq \frac{C\left(\Delta t_{i}\right)^{5}}{\left(1-t_{i}\right)^{4}}
$$

and

$$
(5.62) n^{\frac{1}{4}+p}\left\|k_{0} \sum_{i=1}^{n-1} \widehat{C}_{x t t}\left(\widetilde{t}_{i-1}, \widetilde{S}_{t_{i-1}}\right)\left(S_{t_{i}}-S_{t_{i-1}}\right)\left(\Delta t_{i}\right)^{2}\right\|_{2} \leq C \frac{n^{p} \log n}{n^{\frac{1}{4}}} \underset{n \rightarrow \infty}{\longrightarrow} 0 .
$$

From above, we can conclude about that $n^{\frac{1}{2}+2 p} \mathbf{E}\left(\sup _{t} R_{231}^{n}(t)\right)^{2} \underset{n \rightarrow \infty}{\longrightarrow} 0$.

As for $R_{232}^{n}(t)$, we use the inequality

$$
\left|\Theta_{i}-\Theta_{i}^{1}\right| \leq\left|\Delta K_{t_{i}}^{n}\right|
$$

and we deduce from Definition (2.12) the bound

$$
\left|R_{232}^{n}(t)\right| \leq c \sup _{i}\left|\Delta S_{t_{i}}\right| \int_{0}^{t_{n-1}}\left|\widehat{C}_{x t}\left(t_{u}, S_{u}\right)\right| d u
$$


with

so that

$$
\left|\widehat{C}_{x t}\left(t_{u}, S_{u}\right)\right| \leq \frac{c}{\sqrt{S_{u}}(1-u)}
$$

$$
\left|R_{232}^{n}(t)\right| \leq c \log (n) \sup _{t} S_{t}^{-\frac{1}{2}} \sup _{i}\left|\Delta S_{t_{i}}\right| .
$$

Using the Cauchy-Schwarz inequality, the boundedness of $\mathbf{E} \sup _{t} S_{t}^{-2}$ yields

$$
\mathbf{E} \sup _{t}\left(R_{232}^{n}(t)\right)^{2} \leq C \log ^{2}(n) \sqrt{\mathbf{E} \sup _{i}\left(\Delta S_{t_{i}}\right)^{4}} .
$$

Moreover,

$$
\begin{aligned}
\mathbf{E} \sup _{i}\left(\Delta S_{t_{i}}\right)^{4} & \leq n^{-\frac{3}{2}}+\mathbf{E} \sup _{i}\left(\Delta S_{t_{i}}\right)^{4} \mathbf{1}_{\sup _{i}\left(\Delta S_{t_{i}}\right)^{4} \geq n^{-\frac{3}{2}}} \\
& \leq n^{-\frac{3}{2}}+C \sqrt{\mathbb{P}\left(\sup _{i}\left(\Delta S_{t_{i}}\right)^{4} \geq n^{-\frac{3}{2}}\right)} .
\end{aligned}
$$

By virtue of the Bienaymé-Tchebytchev inequality $\mathbb{P}(|X| \geq k) \leq k^{-8} \mathbf{E} X^{8}$,

$$
\mathbb{P}\left(\sup _{i}\left(\Delta S_{t_{i}}\right)^{4} \geq n^{-\frac{3}{2}}\right) \leq n^{12} \sum_{i} \mathbf{E}\left(\Delta S_{t_{i}}\right)^{32} \leq C n^{-3} .
$$

We deduce that

$$
\mathbf{E} \sup _{i}\left(\Delta S_{t_{i}}\right)^{4} \leq C n^{-\frac{3}{2}}
$$

and finally $\mathbf{E} \sup _{t}\left(R_{232}^{n}(t)\right)^{2} \leq C n^{-3 / 4} \log ^{2}(n)$ so that we can conclude the lemma.

Lemma 5.12. We have

$$
n^{\frac{1}{2}+2 p} \mathbf{E}\left(\sup _{t} R_{24}^{n}(t)\right)^{2} \underset{n \rightarrow \infty}{\longrightarrow} 0 .
$$

Proof. Let us notice that $\sup _{t}\left|R_{24}^{n}(t)\right|$ is bounded by the random variable

$$
k_{0} \sum_{i=1}^{n-1}\left|\widehat{C}_{x}\left(t_{i}, S_{t_{i}}\right)-\widehat{C}_{x}\left(t_{i-1}, S_{t_{i-1}}\right)+\Delta K_{t_{i}}^{n}-\widehat{C}_{x x}\left(t_{i-1}, S_{t_{i-1}}\right)\left(S_{t_{i}}-S_{t_{i-1}}\right)\right| S_{t_{i-1}} .
$$

Using the Ito formula for the increments $\widehat{C}_{x}\left(t_{i}, S_{t_{i}}\right)-\widehat{C}_{x}\left(t_{i-1}, S_{t_{i-1}}\right)$, we obtain

$$
\begin{aligned}
\sup _{t}\left|R_{24}^{n}(t)\right| \leq k_{0} & \sum_{i=1}^{n-1} S_{t_{i-1}} \mid \int_{t_{i-1}}^{t_{i}} \sigma S_{u}\left[\widehat{C}_{x x}\left(u, S_{u}\right)-\widehat{C}_{x x}\left(t_{i-1}, S_{t_{i-1}}\right)\right] d W_{u} \\
& +\frac{1}{2} \int_{t_{i-1}}^{t_{i}} \sigma^{2} S_{u}^{2} \widehat{C}_{x x x}\left(u, S_{u}\right) d u \mid
\end{aligned}
$$

Thus

$$
n^{\frac{1}{4}+p}\left\|\sup _{t} R_{24}^{n}(t)\right\|_{2} \leq T_{n}^{1}+T_{n}^{2}
$$


where

$$
T_{n}^{1}=\sigma k_{0} n^{\frac{1}{4}+p} \sum_{i=1}^{n-1}\left(\int_{t_{i-1}}^{t_{i}} \mathbf{E} S_{t_{i-1}}^{2} S_{u}^{2}\left(\gamma_{u}-\gamma_{t_{i-1}}\right)^{2} d u\right)^{\frac{1}{2}}
$$

and

$$
T_{n}^{2}=\frac{k_{0} n^{\frac{1}{4}+p} \sigma^{4}}{4} \sum_{i=1}^{n-1}\left(\Delta t_{i}\right)^{\frac{1}{2}}\left(\int_{t_{i-1}}^{t_{i}} \mathbf{E} S_{t_{i-1}}^{2} S_{u}^{4} \widehat{C}_{x x x}^{2}\left(u, S_{u}\right) d u\right)^{\frac{1}{2}} .
$$

We first prove that $T_{n}^{1} \underset{n \rightarrow \infty}{\longrightarrow} 0$. Using the Taylor Formula, we get that

$$
\begin{aligned}
\gamma_{u}-\gamma_{t_{i-1}}= & \gamma_{u}-\widehat{C}_{x x}\left(u, S_{t_{i-1}}\right)+\widehat{C}_{x x}\left(u, S_{t_{i-1}}\right)-\gamma_{t_{i-1}} \\
= & \widehat{C}_{x x x}\left(u, S_{t_{i-1}}\right)\left(S_{u}-S_{t_{i-1}}\right)+\frac{1}{2} \widehat{C}_{x x x x}\left(u, \widetilde{S}_{t_{i-1}}\right)\left(S_{u}-S_{t_{i-1}}\right)^{2} \\
& +\widehat{C}_{x x t}\left(\widetilde{t}_{i-1}, S_{t_{i-1}}\right)\left(u-t_{i-1}\right) .
\end{aligned}
$$

Using the suitable estimations from the Appendix, we then obtain

$\left.\mathbf{E} S_{t_{i-1}}^{2} S_{u}^{2}\left(\gamma_{u}-\gamma_{t_{i-1}}\right)\right)^{2} \leq \frac{c \Delta t_{i}}{n^{\frac{7}{8}}\left(1-t_{i}\right)^{\frac{7}{4}}}+\frac{c\left(\Delta t_{i}\right)^{2}}{n^{\frac{3}{2}}\left(1-t_{i}\right)^{3} f^{\prime}\left(t_{i}\right)^{\frac{3}{2}}}+\frac{c\left(\Delta t_{i}\right)^{2}}{n^{3 / 4}\left(1-t_{i}\right)^{1 \frac{1}{4}}}$.

The last estimate follows from Corollary 6.124. Indeed, the proof is the same since $\rho_{\tilde{t}_{i-1}} \leq \rho_{t_{i-1}}$. We can therefore deduce that $T_{n}^{1} \underset{n \rightarrow \infty}{\longrightarrow} 0$.

We then prove that $T_{n}^{2} \underset{n \rightarrow \infty}{\longrightarrow} 0$. We deduce from Appendix the following inequality:

$$
\mathbf{E} S_{t_{i-1}}^{2} S_{u}^{4} \widehat{C}_{x x x}^{2}\left(u, S_{u}\right) \leq \frac{c}{n^{7 / 8}\left(1-t_{i}\right)^{7 / 4}} .
$$

It suffices to obtain the convergence

$$
n^{\frac{1}{4}+p} \sum_{i=1}^{n-1} \frac{\Delta t_{i}}{n^{7 / 16}\left(1-t_{i}\right)^{7 / 8}} \leq \frac{c n^{p}}{n^{3 / 16}} \underset{n \rightarrow \infty}{\longrightarrow} 0
$$

to conclude the lemma.

This last lemma completes the proof of Theorem 5.1.

\subsection{Step 3: Asymptotic distribution.}

From the previous subsection, it turns out that the deviation between the "real world" terminal portfolio and the payoff $h\left(S_{1}\right)$ is essentially composed of a martingale as $n \rightarrow \infty$. To study the asymptotic distribution of $n^{\frac{1}{4}+p} M_{1}^{n}$, we consider it as terminal values of the following sequence of martingales $\left(N_{j}^{n}\right)_{j=0, \cdots, n}$ with respect to the filtration $\mathcal{F}^{n}=\left(\mathcal{F}_{t_{i}}\right)_{i}$ :

$$
N_{j}^{n}:=n^{\frac{1}{4}+p} M_{j}^{n}=\sum_{i=1}^{j}\left(\chi_{i}+\vartheta_{i}\right)
$$


where

$$
\begin{aligned}
\chi_{i} & :=k_{0} n^{1 / 4+p} \gamma_{t_{i-1}} S_{t_{i-1}}^{2}\left[\mathcal{E}_{t_{i-1}}^{t_{i}}\right]_{c}, \\
\vartheta_{i} & :=k_{0} n^{1 / 4+p} K_{t_{i-1}}^{n}\left(S_{t_{i}}-S_{t_{i-1}}\right) .
\end{aligned}
$$

Theorem 5.13. The following convergence holds:

$$
N_{1}^{n} \underset{n \rightarrow \infty}{\stackrel{d}{\longrightarrow}} Z
$$

where the law of $Z$ is given by the characteristic function $\phi_{Z}(s)=\mathbf{E} e^{-\frac{s^{2}}{2} \eta^{2}}$ with

$$
\begin{aligned}
\eta^{2}:=\mathcal{Q}(\mu)\left(k_{0} \sigma\right)^{1-4 p} S_{1}^{2} \times & \\
& \int_{0}^{\infty} x^{4 p}\left\{\left(\int_{x}^{\infty} J\left(y, S_{1}\right) d y\right)^{2}+\left(1-\frac{2}{\pi}\right) \widetilde{J}\left(x, S_{1}\right)^{2}\right\} d x,
\end{aligned}
$$

and

$$
\begin{aligned}
J\left(x, S_{1}\right) & :=\frac{1}{2 x} \int_{-\infty}^{\infty} h^{\prime}\left(S_{u} e^{\sqrt{x} y+x / 2}\right)\left(-y^{2}-\sqrt{x} y+1\right) \varphi(y) d y \\
\widetilde{J}\left(x, S_{1}\right) & :=\frac{1}{\sqrt{x}} \int_{-\infty}^{\infty} h^{\prime}\left(S_{u} e^{\sqrt{x} y+x / 2}\right) y \varphi(y) d y .
\end{aligned}
$$

Moreover

$$
\mathbf{E}\left(N_{1}^{n}\right)^{2} \underset{n \rightarrow \infty}{\longrightarrow} \mathbf{E} \eta^{2}
$$

We achieve the proof of this theorem by means of the result page 58 in [7] recalled by Theorem 6.1 in the Appendix. (Note that the completion of the $\sigma$-field generated by the collection $\left(\mathcal{F}^{n}\right)_{n}$ coincides with $\mathcal{F}_{1}$.) We thus need to prove the following lemmas.

Lemma 5.14. The sequence of martingales $\left(N_{i}^{n}\right)_{i=0, \cdots, n}$ satisfies

$$
\text { for all } \varepsilon>0, \quad \sum_{i} \mathbf{E}\left(\left(\chi_{i}+\vartheta_{i}\right)^{2} \mathbf{1}_{\left|\chi_{i}+\vartheta_{i}\right|>\varepsilon} \mid \mathcal{F}_{t_{i-1}}\right) \underset{n \rightarrow \infty}{\stackrel{P}{\longrightarrow}} 0 \text {. }
$$

Proof. We use the inequality $\left(\chi_{i}+\vartheta_{i}\right)^{2} \leq 2 \chi_{i}^{2}+2 \vartheta_{i}^{2}$ and we shall prove a stronger result, i.e. a convergence in $L^{1}$.

First let us show that

$$
\mathbf{E}\left(\vartheta_{i}^{2} \mathbf{1}_{\left|\chi_{i}+\vartheta_{i}\right|>\varepsilon}\right) \underset{n \rightarrow \infty}{\longrightarrow} 0
$$

By virtue of the Markov inequality, we obtain

$$
\begin{aligned}
\mathbf{E}\left(\vartheta_{i}^{2} \mathbf{1}_{\left|\vartheta_{i}\right|>\varepsilon / 2}\right) & \leq \sqrt{\mathbf{E} \vartheta_{i}^{4}} \sqrt{\mathbb{P}\left(\left|\vartheta_{i}\right|>\varepsilon / 2\right)} \\
& \leq C \varepsilon^{-6} \sqrt{\mathbf{E} \vartheta_{i}^{4}} \sqrt{\mathbf{E} \vartheta_{i}^{12}}
\end{aligned}
$$


By independance, we have

$$
\begin{aligned}
\mathbf{E} \vartheta_{i}^{4} & =k_{0}^{2} n^{1+4 p} \mathbf{E}\left(K_{t_{i-1}}^{n}\right)^{4} S_{t_{i-1}}^{4} \mathbf{E}\left(\mathcal{E}_{t_{i-1}}^{t_{i}}\right)^{4} \\
\mathbf{E} \vartheta_{i}^{12} & =k_{0}^{2} n^{3+12 p} \mathbf{E}\left(K_{t_{i-1}}^{n}\right)^{12} S_{t_{i-1}}^{12} \mathbf{E}\left(\mathcal{E}_{t_{i-1}}^{t_{i}}\right)^{12}
\end{aligned}
$$

Recall that

$$
K_{t_{i-1}}^{n}=-\int_{0}^{t_{i-1}} \widehat{C}_{x t}\left(u, S_{u}\right) d u
$$

and by virtue of Lemma 6.8 there exists a constant $C$ such that

$$
\left|K_{t_{i-1}}^{n}\right|^{4} \leq C \sup _{0 \leq u \leq T} S_{u}^{-2}\left(\int_{0}^{t_{n-1}} \frac{d u}{1-u}\right)^{4} \leq C \sup _{0 \leq u \leq T} S_{u}^{-2} \log ^{4}(n) .
$$

We deduce that

$$
\begin{aligned}
\mathbf{E} \vartheta_{i}^{4} & \leq C \log ^{4}(n) n^{4 p-1} \\
\mathbf{E} \vartheta_{i}^{12} & \leq C \log ^{12}(n) n^{12 p-3}
\end{aligned}
$$

Since $p<1 / 8$, we deduce

$$
\begin{aligned}
\sum_{i} \mathbf{E}\left(\vartheta_{i}^{2} \mathbf{1}_{\left|\vartheta_{i}\right|>\varepsilon / 2}\right) & \leq C \varepsilon^{-6} n^{8 p-1} \log ^{8}(n) \sum_{i \leq n} n^{-1} \\
& \leq C \varepsilon^{-6} n^{8 p-1} \log ^{8}(n) \underset{n \rightarrow \infty}{\longrightarrow} 0 .
\end{aligned}
$$

Let us study $\mathbf{E}\left(\vartheta_{i}^{2} \mathbf{1}_{\left|\chi_{i}\right|>\varepsilon / 2}\right)$. Again,

$$
\begin{aligned}
\mathbf{E}\left(\vartheta_{i}^{2} \mathbf{1}_{\left|\chi_{i}\right|>\varepsilon / 2}\right) & \leq \sqrt{\mathbf{E} \vartheta_{i}^{4}} \sqrt{\mathbb{P}\left(\left|\chi_{i}\right|>\varepsilon / 2\right)} \\
& \leq C \varepsilon^{-2} \sqrt{\mathbf{E} \vartheta_{i}^{4}} \sqrt{\mathbf{E} \chi_{i}^{4}} .
\end{aligned}
$$

Once again by independence,

$$
\mathbf{E} \chi_{i}^{4}=k_{0}^{4} n^{1+4 p} \mathbf{E} \gamma_{t_{i-1}}^{4} S_{t_{i-1}}^{8} \mathbf{E}\left[\mathcal{E}_{t_{i-1}}^{t_{i}}\right]_{c}^{4} .
$$

We easily deduce from Lemma 4.1 the inequality

$$
\mathbf{E}\left[\mathcal{E}_{t_{i-1}}^{t_{i}}\right]_{c}^{4} \leq C\left(\Delta t_{i}\right)^{2} .
$$

Using Corollary 6.119 we obtain

$$
\mathbf{E} \chi_{i}^{4} \leq C n^{1+4 p} \frac{\left(\Delta t_{i}\right)^{2}}{\left(n^{1 / 4} \sqrt{1-t_{i-1}}\right)^{3}} \leq C n^{4 p-1 / 4} \text {. }
$$

Since $p<\frac{1}{16}<\frac{3}{32}$, then

$$
\begin{aligned}
\sum_{i} \mathbf{E}\left(\vartheta_{i}^{2} \mathbf{1}_{\left|\chi_{i}\right|>\varepsilon / 2}\right) & \leq C \varepsilon^{-2} n^{4 p} \log ^{2}(n) \sum_{i} \frac{\Delta t_{i}}{n^{3 / 8}\left(1-t_{i-1}\right)^{3 / 4}} \\
& \leq C \varepsilon^{-2} n^{4 p-3 / 8} \log ^{2}(n) \sum_{i} \frac{\Delta t_{i}}{1-t_{i-1}} \\
& \leq C \varepsilon^{-2} n^{-3 / 8+4 p} \log ^{3}(n) \underset{n \rightarrow \infty}{\longrightarrow} 0
\end{aligned}
$$


From the inequality $\mathbf{1}_{\left|\chi_{i}+\vartheta_{i}\right|>\varepsilon} \leq \mathbf{1}_{\left|\chi_{i}\right|>\varepsilon / 2}+\mathbf{1}_{\left|\vartheta_{i}\right|>\varepsilon / 2}$ we then deduce that

$$
\sum_{i} \mathbf{E}\left(\vartheta_{i}^{2} \mathbf{1}_{\left|\chi_{i}+\vartheta_{i}\right|}>\varepsilon\right) \underset{n \rightarrow \infty}{\longrightarrow} 0
$$

Second let us show that

$$
\mathbf{E}\left(\chi_{i}^{2} \mathbf{1}_{\left|\chi_{i}+\vartheta_{i}\right|>\varepsilon}\right) \underset{n \rightarrow \infty}{\longrightarrow} 0 .
$$

In the same way, we have

$$
\begin{aligned}
\mathbf{E}\left(\chi_{i}^{2} \mathbf{1}_{\left|\vartheta_{i}\right|>\varepsilon / 2}\right) & \leq \sqrt{\mathbf{E} \chi_{i}^{4}} \sqrt{\mathbb{P}\left(\left|\vartheta_{i}\right|>\varepsilon / 2\right)} \\
& \leq C \varepsilon^{-6} \sqrt{\mathbf{E} \chi_{i}^{4}} \sqrt{\mathbf{E} \vartheta_{i}^{12}} .
\end{aligned}
$$

From (5.73) we have

$$
\mathbf{E} \chi_{i}^{4} \leq C n^{4 p-1 / 4}
$$

Thus, using $p<\frac{1}{16}<\frac{5}{64}$,

$$
\sum_{i} \mathbf{E}\left(\chi_{i}^{2} \mathbf{1}_{\left|\vartheta_{i}\right|>\varepsilon / 2}\right) \leq C \varepsilon^{-6} n^{8 p-5 / 8} \log ^{6}(n) \underset{n \rightarrow \infty}{\longrightarrow} 0 .
$$

Let us now study $\mathbf{E}\left(\chi_{i}^{2} \mathbf{1}_{\left|\chi_{i}\right|>\varepsilon / 2}\right)$.

$$
\begin{aligned}
\mathbf{E}\left(\chi_{i}^{2} \mathbf{1}_{\left|\chi_{i}\right|>\varepsilon / 2}\right) & \leq \sqrt{\mathbf{E} \chi_{i}^{4}} \sqrt{\mathbb{P}\left(\left|\chi_{i}\right|>\varepsilon / 2\right)} \\
& \leq C \varepsilon^{-2} \mathbf{E} \chi_{i}^{4} .
\end{aligned}
$$

Using the bound (5.73), we obtain

$$
\sum_{i} \mathbf{E}\left(\chi_{i}^{2} \mathbf{1}_{\left|\chi_{i}\right|>\varepsilon / 2}\right) \leq C n^{4 p-1 / 4} \underset{n \rightarrow \infty}{\longrightarrow} 0
$$

We finally conclude the lemma.

Lemma 5.15. The sequence of martingales $\left(M_{i}^{n}\right)_{i=0, \cdots, n}$ satisfies the following convergence

$$
V_{n}^{2}:=\sum_{i} \mathbf{E}\left(\left(\chi_{i}+\vartheta_{i}\right)^{2} \mid \mathcal{F}_{t_{i-1}}\right) \underset{n \rightarrow \infty}{\stackrel{d}{\longrightarrow}} \eta^{2}
$$

where

$$
\begin{aligned}
\eta:=\mathcal{Q}(\mu)\left(k_{0} \sigma\right)^{1-4 p} S_{1}^{2} \times \\
\quad \int_{0}^{\infty} x^{4 p}\left\{\left(\int_{x}^{\infty} J\left(y, S_{1}\right) d y\right)^{2}+\left(1-\frac{2}{\pi}\right) \widetilde{J}\left(x, S_{1}\right)^{2}\right\} d x
\end{aligned}
$$


with

$$
\begin{aligned}
J\left(x, S_{1}\right) & :=\frac{1}{2 x} \int_{-\infty}^{\infty} h^{\prime}\left(S_{u} e^{\sqrt{x} y+x / 2}\right)\left(-y^{2}-\sqrt{x} y+1\right) \varphi(y) d y \\
\widetilde{J}\left(x, S_{1}\right) & :=\frac{1}{\sqrt{x}} \int_{-\infty}^{\infty} h^{\prime}\left(S_{u} e^{\sqrt{x} y+x / 2}\right) y \varphi(y) d y .
\end{aligned}
$$

Proof. First, let us study the term

$$
\xi_{n}^{\vartheta}:=\sum_{i} \mathbf{E}\left(\vartheta_{i}^{2} \mid \mathcal{F}_{t_{i-1}}\right)
$$

By independence, we obtain

$$
\mathbf{E}\left(\vartheta_{i}^{2} \mid \mathcal{F}_{t_{i-1}}\right)=k_{0}^{2} n^{1 / 2+2 p}\left(K_{t_{i-1}}^{n}\right)^{2} S_{t_{i-1}}^{2} \mathbf{E}\left(\mathcal{E}_{t_{i-1}}^{t_{i}}\right)^{2} .
$$

Hence, using Lemma 4.1 and the change of variable $y=\rho_{u}^{2}$ and $x_{i}=\rho_{t_{i}}^{2}$,

$$
\begin{aligned}
\mathbf{E}\left(\vartheta_{i}^{2} \mid \mathcal{F}_{t_{i-1}}\right) & =k_{0}^{2} n^{1 / 2+2 p} K_{t_{i-1}}^{n 2} S_{t_{i-1}}^{2} \sigma^{2} \Delta t_{i}\left(1+O\left(n^{-1}\right)\right) \\
& =k_{0}^{2} \sigma^{2} n^{1 / 2+2 p} S_{t_{i-1}}^{2}\left(\int_{0}^{t_{i-1}} \widehat{C}_{x t}\left(u, S_{u}\right) d u\right)^{2} \Delta t_{i}\left(1+O\left(n^{-1}\right)\right) \\
& =k_{0}^{2} \sigma^{2} S_{t_{i-1}}^{2}\left(\int_{0}^{t_{i-1}} \widehat{C}_{x t}\left(u, S_{u}\right) d u\right)^{2} \frac{n^{1 / 2+2 p} \Delta t_{i}}{x_{i-1}-x_{i}} \Delta x_{i}\left(1+O\left(n^{-1}\right)\right) \\
& =k_{0}^{2} \sigma^{2} S_{t_{i-1}}^{2}\left(\int_{x_{i-1}}^{\rho_{0}^{2}} \widehat{C}_{x t}\left(u, S_{u}\right) \widehat{\sigma}_{u}^{-2} d y\right)^{2} \frac{n^{1 / 2+2 p} \Delta t_{i}}{x_{i-1}-x_{i}} \Delta x_{i}\left(1+O\left(n^{-1}\right)\right) .
\end{aligned}
$$

We then deduce that

$$
\xi_{n}^{\vartheta}=\left(1+O\left(n^{-1}\right)\right) \int_{0}^{\infty} z_{n}^{\vartheta}(x) d x
$$

where

$$
z_{n}^{\vartheta}(x):=S_{t_{i-1}}^{2} k_{0}^{2} \sigma^{2} \sum_{i}\left(\int_{x_{i-1}}^{\rho_{0}^{2}} \widehat{C}_{x t}\left(u, S_{u}\right) \widehat{\sigma}_{u}^{-2} d x\right)^{2} \frac{n^{1 / 2+2 p} \Delta t_{i}}{x_{i-1}-x_{i}} \mathbf{1}_{\left(x_{i}, x_{i-1}\right]}(x) .
$$

Recall that

$$
\left|\widehat{C}_{x t}\left(u, S_{u}\right) \widehat{\sigma}_{u}^{-2}\right| d u \leq c G_{1}\left(x, S_{u}\right), \quad x=\rho_{u}^{2}
$$

where

$$
G_{1}(x, y)=\frac{1}{x} e^{-x / 8}\left(\sum_{j=1}^{p} \frac{\left|\log \left(y / K_{j}\right)\right|}{\sqrt{x}} \exp \left\{-\frac{\log ^{2}\left(y / K_{j}\right)}{2 x}\right\}+\sqrt{x}+x\right) .
$$

In particular,

$$
\sqrt{x} G_{1}(x, y) \leq G(x)
$$

where $G(x)=c x^{-\frac{1}{2}} e^{-x / 16}, c>0$ is a constant. Hence, a.s.

$$
\left|\int_{x_{i-1}}^{\rho_{0}^{2}} \widehat{C}_{x t}\left(u, S_{u}\right) \widehat{\sigma}_{u}^{-2} d y\right| \leq \int_{x}^{\infty} G\left(x^{\prime}\right) d x^{\prime} \leq \int_{0}^{\infty} G\left(x^{\prime}\right) d x^{\prime}<+\infty .
$$


Therefore, using (4.34),

$$
\left|z_{n}^{\vartheta}(x)\right| \leq C(1+x)\left(\int_{x}^{\infty} G\left(x^{\prime}\right) d x^{\prime}\right)^{2} \sup _{u \in[0,1]} S_{u}^{2}
$$

But, due to Hölder's inequality,

$$
\int_{0}^{\infty}(1+x)\left(\int_{x}^{\infty} G\left(x^{\prime}\right) d x^{\prime}\right)^{2} d x<+\infty .
$$

We can thus apply Lebesgue's theorem using Corollary 6.22 and (4.33):

$$
\xi_{n}^{\vartheta} \underset{n \rightarrow \infty}{\stackrel{a . s .}{\longrightarrow}} \mathcal{Q}(\mu)\left(k_{0} \sigma\right)^{1-4 p} S_{1}^{2} \int_{0}^{\infty} x^{4 p}\left(\int_{x}^{\infty} J\left(y, S_{1}\right) d y\right)^{2} d x .
$$

Second, let us study the term

$$
\xi_{n}^{\chi}=\sum_{i} \mathbf{E}\left(\chi_{i}^{2} \mid \mathcal{F}_{t_{i-1}}\right) .
$$

By independence, we obtain

$$
\mathbf{E}\left(\chi_{i}^{2} \mid \mathcal{F}_{t_{i-1}}\right)=k_{0}^{2} n^{1 / 2+2 p} \gamma_{t_{i-1}}^{2} S_{t_{i-1}}^{4} \mathbf{E}\left[\mathcal{E}_{t_{i-1}}^{t_{i}}\right]_{c}^{2} .
$$

Then

$$
\mathbf{E}\left(\chi_{i}^{2} \mid \mathcal{F}_{t_{i-1}}\right)=k_{0}^{2} \sigma^{2} n^{1 / 2+2 p} \gamma_{t_{i-1}}^{2} S_{t_{i-1}}^{4}\left(1-\frac{2}{\pi}\right) \Delta t_{i}(1+o(1)) .
$$

We then deduce that

$$
\sum_{i} \mathbf{E}\left(\chi_{i}^{2} \mid \mathcal{F}_{t_{i-1}}\right)=\left(1+O\left(n^{-1}\right)\right) \int_{0}^{\infty} z_{n}^{\chi}(x) d x
$$

where

$$
z_{n}^{\chi}(x):=S_{t_{i-1}}^{4} k_{0}^{2} \sigma^{2} \sum_{i} \gamma_{t_{i-1}}^{2} \frac{n^{1 / 2+2 p} \Delta t_{i}}{x_{i-1}-x_{i}} \mathbf{1}_{\left(x_{i}, x_{i-1}\right]}(x) .
$$

Let us obtain a suitable bound for $z_{n}^{\chi}(x)$, integrable in $x$. Recall that

$$
\begin{aligned}
\gamma_{t_{i-1}} & =\widehat{C}_{x x}\left(t_{i-1}, S_{t_{i-1}}\right) \\
& =\frac{1}{\rho_{t_{i-1}} S_{t_{i-1}}} \int_{-\infty}^{\infty} h^{\prime}\left(S_{t_{i-1}} e^{\rho_{t_{i-1}} y+\rho_{t_{i-1}}^{2} / 2}\right) y \varphi(y) d y \\
& =\frac{1}{\sqrt{x_{i-1}} S_{t_{i-1}}} \int_{-\infty}^{\infty} h^{\prime}\left(S_{t_{i-1}} e^{\sqrt{x_{i-1}} y+x_{i-1} / 2}\right) y \varphi(y) d y .
\end{aligned}
$$

Due to Inequality (6.117), we claim that a.s. $(\omega)$ for $n$ large enough, there is a constant $c_{\omega}$ which does not depend on $n$ such that

$$
\left|\gamma_{t_{i-1}}\right| \leq C \sup _{u \leq 1} S_{u}^{-3 / 2} e^{-x / 8}\left(\mathbf{1}_{x \geq 1}+\left(\frac{e^{-\frac{c_{\omega}}{x}}}{\sqrt{x}}+1\right) \mathbf{1}_{x \leq 1}\right) .
$$


Indeed, this is obvious for $x \geq 1$. Otherwise, $1 \geq x=\rho_{u}^{2} \geq c n^{1 / 2}\left(1-u^{n}(x)\right)$ implies that $u=u^{n}(x)$ is close to 1 uniformly in $x \leq 1$ as soon as $n$ is large enough. It then suffices to choose $S_{1}$ out of the null-set $\left\{S_{1}=K_{1}, \cdots, K_{p}\right\}$ to obtain by continuity that $S_{u^{n}(x)}$ is also far enough from the points $K_{1}, \cdots, K_{p}$ if $x \geq 1$.

We conclude that for all $j, \log ^{2}\left(K_{j} / S_{u^{n}(x)}\right) \geq c_{\omega, j}$ for some constants $c_{\omega, j}>0$.

Therefore,

$$
\left|S_{t_{i-1}}\right|^{4}\left|\gamma_{t_{i-1}}\right|^{2} \leq C \sup _{0 \leq u \leq 1} S_{u}^{4} \sup _{0 \leq u \leq 1} S_{u}^{-3} e^{-x / 4}\left(\mathbf{1}_{x \geq 1}+\left(\frac{e^{-\frac{c_{\omega}}{x}}}{\sqrt{x}}+1\right)^{2} \mathbf{1}_{x \leq 1}\right) .
$$

Thus, due to (4.33)

$$
\left|z_{n}^{\chi}(x)\right| \leq C \sup _{0 \leq u \leq 1} S_{u}^{4} \sup _{0 \leq u \leq 1} S_{u}^{-3}(1+x) e^{-x / 4}\left(\mathbf{1}_{x \geq 1}+\left(\frac{e^{-\frac{c_{\omega}}{x}}}{\sqrt{x}}+1\right)^{2} \mathbf{1}_{x \leq 1}\right) .
$$

We can then apply the dominated convergence theorem using the limit (4.34). We obtain

$$
\xi_{n}^{\chi} \underset{n \rightarrow \infty}{\stackrel{a . s .}{\longrightarrow}}\left(1-\frac{2}{\pi}\right) Q(\mu)\left(k_{0} \sigma\right)^{1-4 p} S_{1}^{2} \int_{0}^{\infty} x^{4 p} \widetilde{J}\left(x, S_{1}\right)^{2} d x
$$

Finally, let us study the term $\sum_{i} \mathbf{E}\left(\chi_{i} \vartheta_{i} \mid \mathcal{F}_{t_{i-1}}\right)$.

By independence, we have

$$
\mathbf{E}\left(\chi_{i} \vartheta_{i} \mid \mathcal{F}_{t_{i-1}}\right)=k_{0}^{2} n^{1 / 2+2 p} \gamma_{t_{i-1}} S_{t_{i-1}}^{2} K_{t_{i-1}}^{n} S_{t_{i-1}} \mathbf{E}\left(\left[\mathcal{E}_{t_{i-1}}^{t_{i}}\right]_{c} \mathcal{E}_{t_{i-1}}^{t_{i}}\right) .
$$

But

$$
\mathbf{E}\left(\left[\mathcal{E}_{t_{i-1}}^{t_{i}}\right]_{c} \mathcal{E}_{t_{i-1}}^{t_{i}}\right)=\mathbf{E}\left[\mathcal{E}_{t_{i-1}}^{t_{i}}\right]_{c}^{2} \operatorname{sgn} \mathcal{E}_{t_{i-1}}^{t_{i}}=\left(1-\frac{2}{\pi}\right) \sigma^{2}\left(\Delta t_{i}\right)^{\frac{3}{2}}(1+o(1)) .
$$

Due to (4.33), we obtain

$$
\frac{\left(\Delta t_{i}\right)^{\frac{3}{2}} n^{1 / 2+2 p}}{x_{i-1}-x_{i}} \underset{n \rightarrow \infty}{\longrightarrow} 0
$$

From the bounds (4.34), (5.88), (5.99) and by applying again Lebesgue's theorem, we then deduce the following limit

$$
\sum_{i} \mathbf{E}\left(\chi_{i} \vartheta_{i} \mid \mathcal{F}_{t_{i-1}}\right) \underset{n \rightarrow \infty}{\stackrel{a . s .}{\longrightarrow}} 0
$$


Lemma 5.16. We have

$$
\mathbf{E}\left(N_{1}^{n}\right)^{2} \underset{n \rightarrow \infty}{\longrightarrow} \mathbf{E} \eta^{2} .
$$

Proof. Due to the independence of the increments of the Wiener process, we have $\mathbf{E}\left(\chi_{i}+\vartheta_{i}\right)\left(\chi_{j}+\vartheta_{j}\right)=0$ whenever $i \neq j$. We thus obtain

$$
\begin{aligned}
\mathbf{E}\left(N_{1}^{n}\right)^{2} & =\sum_{i} \mathbf{E}\left(\chi_{i}+\vartheta_{i}\right)^{2} \\
& =\mathbf{E} \sum_{i} \mathbf{E}\left(\left(\chi_{i}+\vartheta_{i}\right)^{2} \mid \mathcal{F}_{t_{i-1}}\right)
\end{aligned}
$$

But

$$
\sum_{i} \mathbf{E}\left(\left(\chi_{i}+\vartheta_{i}\right)^{2} \mid \mathcal{F}_{t_{i-1}}\right) \leq 2 \sum_{i} \mathbf{E}\left(\chi_{i}^{2}+\vartheta_{i}^{2} \mid \mathcal{F}_{t_{i-1}}\right)=2\left(\xi_{n}^{\chi}+\xi_{n}^{\vartheta}\right)
$$

Let us show that $\xi_{n}:=\xi_{n}^{\chi}+\xi_{n}^{\vartheta}$ is uniformly integrable. First let us note that $\xi_{n}$ is bounded in $L^{1}(\Omega)$. Indeed, from Corollary 6.5, Inequalities (5.89) and (4.33), we obtain for all $n$

$$
\mathbf{E}\left|\xi_{n}\right| \leq C \int_{0}^{\infty}(1+x)\left(\left(\mathbf{E} S_{1}^{2}\right) \frac{e^{-x}}{\sqrt{x}}+\left(\int_{x}^{\infty} G\left(x^{\prime}\right) d x^{\prime}\right)\right)<\infty
$$

Now, using the Cauchy-Schwarz inequality and then the Markov inequality, we have

$$
\begin{aligned}
\mathbf{E} \xi_{n}^{\vartheta} \mathbf{1}_{\xi_{n} \geq k} & \leq C \int_{0}^{\infty}(1+x)\left(\int_{x}^{\infty} G\left(x^{\prime}\right) d x^{\prime}\right)^{2} d x \sqrt{\mathbf{E} S_{1}^{4}} \sqrt{\mathbb{P}\left(\xi_{n} \geq k\right)} \\
& \leq C \sqrt{\frac{\sup _{n} \mathbf{E}\left|\xi_{n}\right|}{k}} \underset{k \rightarrow \infty}{\longrightarrow} 0 .
\end{aligned}
$$

Recall that

$$
z_{n}^{\chi}(x) \mathbf{1}_{\xi^{n} \geq M_{0}}:=k_{0}^{2} \sigma^{2} \sum_{i} S_{t_{i-1}}^{4} \gamma_{t_{i-1}}^{2} \mathbf{1}_{\xi^{n} \geq M_{0}} \frac{n^{1 / 2+2 p} \Delta t_{i}}{x_{i-1}-x_{i}} \mathbf{1}_{\left(x_{i}, x_{i-1}\right]}(x) .
$$

Therefore, applying successively the Cauchy-Schwarz inequality, (4.33), Corollary 6.16 , and the Markov inequality, we obtain

$$
\begin{aligned}
\mathbf{E} \xi_{n}^{\chi} \mathbf{1}_{\xi_{n} \geq k} & \leq C \sum_{i}\left(\mathbf{E} S_{t_{i-1}}^{5} \gamma_{t_{i-1}}^{5 / 2}\right)^{4 / 5}\left(\mathbb{P}\left(\xi^{n} \geq k\right)\right)^{1 / 5} \frac{n^{1 / 2+2 p} \Delta t_{i}}{x_{i-1}-x_{i}} \mathbf{1}_{\left(x_{i}, x_{i-1}\right]}(x) \\
& \leq C \int_{0}^{\infty}(1+x)\left(\frac{e^{-5 x / 32}}{x^{15 / 16}}\right)^{4 / 5} d x\left(\frac{\sup _{n} \mathbf{E} \xi_{n}}{k}\right)^{1 / 5} \underset{k \rightarrow \infty}{\longrightarrow} 0 .
\end{aligned}
$$

Therefore, $\xi_{n}$ is uniformly integrable, and so is $\sum_{i} \mathbf{E}\left(\left(\chi_{i}+\vartheta_{i}\right)^{2} \mid \mathcal{F}_{t_{i-1}}\right)$, which moreover converges to $\eta$ a.s. This yields the conclusion of the Lemma.

This last lemma completes the proof of Theorem 5.13. 
5.4. Conclusion. Let us summarize the results of the previous theorems:

$$
\begin{array}{r}
n^{\frac{1}{2}+2 p} \mathbf{E}\left(\varepsilon_{t}^{n}\right)^{2} \underset{n \rightarrow \infty}{\longrightarrow} 0 \\
n^{\frac{1}{4}+p} N_{1}^{n} \underset{n \rightarrow \infty}{\stackrel{d}{\longrightarrow}} Z .
\end{array}
$$

Therefore

$$
n^{\frac{1}{4}+p}\left(V_{1}^{n}-h\left(S_{1}\right)\right) \underset{n \rightarrow \infty}{\stackrel{d}{\longrightarrow}} Z
$$

and

$$
n^{\frac{1}{2}+2 p} \mathbf{E}\left(V_{1}^{n}-h\left(S_{1}\right)\right)^{2} \underset{n \rightarrow \infty}{\longrightarrow} \mathbf{E} \eta^{2}=\mathbf{E} Z^{2} .
$$

The proof of the limit theorem is then complete. 


\section{Appendix}

The following limit result combines Theorem 3.2 page 58 [7] with its corollary 3.1 and its remarks.

Theorem 6.1 ([7] page 58). Let $\left\{M_{i}^{n}, \mathcal{F}_{t_{i}}, 0 \leq i \leq n\right\}$ be a zero-mean square integrable martingale with increments $\Delta M_{i}^{n}=X_{i}^{n}$ and let $\eta^{2}$ be an a.s. finite r.v. Suppose that

$$
\begin{aligned}
& \text { for all } \varepsilon>0, \quad \sum_{i} \mathbf{E}\left(\left(X_{i}^{n}\right)^{2} \mathbf{1}_{\left|\chi_{i}^{n}\right|>\varepsilon} \mid \mathcal{F}_{t_{i-1}}\right) \underset{n \rightarrow \infty}{\stackrel{P}{\longrightarrow}} 0, \\
& V_{n}^{2}=\sum_{i} \mathbf{E}\left(\left(X_{i}^{n}\right)^{2} \mid \mathcal{F}_{t_{i-1}}\right) \underset{n \rightarrow \infty}{\stackrel{P}{\longrightarrow}} \eta^{2} .
\end{aligned}
$$

Then $M_{n}^{n} \underset{n \rightarrow \infty}{\stackrel{d}{\longrightarrow}} Y$ where the r.v. $Y$ has the characteristic function $\mathbf{E} \exp -\frac{1}{2} \eta^{2} t^{2}$.

\subsection{A0.}

We recall from [3] the following expressions for the successive derivatives. They are based on direct computations using the integration by parts formula under suitable assumptions on the payoff function $h$.

Lemma 6.2. Let $\widehat{C}(t, x)$ is given by (2.7). Then

$$
\begin{aligned}
\widehat{C}_{x}(t, x) & =\int_{-\infty}^{\infty} h^{\prime}\left(x e^{\rho y+\rho^{2} / 2}\right) \varphi(y) d y \\
\widehat{C}_{x x}(t, x) & =\frac{1}{\rho x} \int_{-\infty}^{\infty} h^{\prime}\left(x e^{\rho y+\rho^{2} / 2}\right) y \varphi(y) d y \\
\widehat{C}_{x x x}(t, x) & \left.=\frac{1}{\rho^{2} x^{2}} \int_{-\infty}^{\infty} h^{\prime} x e^{\rho y+\rho^{2} / 2}\right) P_{2}(y) \varphi(y) d y \\
\widehat{C}_{x x x x}(t, x) & =\frac{1}{\rho^{3} x^{3}} \int_{-\infty}^{\infty} h^{\prime}\left(x e^{\rho y+\rho^{2} / 2}\right) P_{3}(y) \varphi(y) d y
\end{aligned}
$$

where

$$
\begin{aligned}
& P_{2}(y):=y^{2}-\rho y-1 \\
& P_{3}(y):=y^{3}-3 \rho y^{2}+\left(2 \rho^{2}-3\right) y+3 \rho .
\end{aligned}
$$

In particular, $\left|\widehat{C}_{x}(t, x)\right| \leq\left\|h^{\prime}\right\|_{\infty}$.

Similarly, we obtain the following expressions for the successive derivatives in $t$ : 
Lemma 6.3. Let $\widehat{C}(t, x)$ is given by (2.7). Then

$$
\begin{aligned}
\widehat{C}_{t}(t, x) & =\frac{-\widehat{\sigma}_{t}^{2} x}{2 \rho} \int_{-\infty}^{\infty} h^{\prime}\left(x e^{\rho y+\rho^{2} / 2}\right) y \varphi(y) d y \\
\widehat{C}_{t x}(t, x) & =\frac{\widehat{\sigma}_{t}^{2}}{2 \rho^{2}} \int_{-\infty}^{\infty} h^{\prime}\left(x e^{\rho y+\rho^{2} / 2}\right) Q_{2}(y) \varphi(y) d y \\
\widehat{C}_{x x t}(t, x) & =\frac{\widehat{\sigma}_{t}^{2}}{2 \rho^{3} x} \int_{-\infty}^{\infty} h^{\prime}\left(x e^{\rho y+\rho^{2} / 2}\right) Q_{3}(y) \varphi(y) d y
\end{aligned}
$$

with

$$
\begin{aligned}
& Q_{2}(y):=-y^{2}-\rho y+1 \\
& Q_{3}(y):=-y^{3}-\rho y^{2}+3 y+\rho .
\end{aligned}
$$

To study the residual terms generated by the discretization of the theoretical $\widehat{C}\left(T, S_{T}\right)$, we use Taylor approximations. We then need to estimate some bounds of the successive derivatives of $\widehat{C}$.

Lemma 6.4. There is a constant $C>0$ such that

$$
\left|\widehat{C}_{x x}(t, x)\right| \leq C \frac{e^{-\rho^{2} / 8}}{\rho x^{3 / 2}} \sum_{j=1}^{p} \exp \left\{-\frac{1}{2} \frac{\log ^{2}\left(K_{j} / x\right)}{\rho^{2}}\right\}+c \frac{e^{-\rho^{2} / 8}}{x^{3 / 2}}
$$

Corollary 6.5. There exists a constant $C$ such that for $t \in[0,1[$

$$
\mathbf{E} S_{t}^{4} \widehat{C}_{x x}^{2}\left(t, S_{t}\right) \leq \frac{C}{\rho} e^{-\rho^{2} / 4}
$$

Similarly, we can deduce the following bounds:

Corollary 6.6. There exists a constant $c$ such that for $t \in[0,1[$

$$
\mathbf{E} S_{t}^{2} \widehat{C}_{x x}^{2}\left(t, S_{t}\right) \leq c\left(\sum_{j=1}^{p} \frac{1}{\rho^{2} \sqrt{2 u^{2}+1}} \exp \left\{-\frac{v_{j}^{2}}{2 u^{2}+1}\right\}+e^{-\rho^{2} / 4}\right)
$$

where $c$ is a constant, $u=\alpha_{t} / \rho$ and

$$
v_{j}:=\frac{\log \left(S_{0} / K_{j}\right)-\alpha_{t}^{2} / 2}{\rho}+\frac{\rho}{2}
$$

Corollary 6.7. There exists a constant $c$ such that for $t \in\left[\frac{1}{2}, 1[\right.$,

$$
\mathbf{E} S_{t}^{2} \widehat{C}_{x x}^{2}\left(t, S_{t}\right) \leq c\left(\frac{1}{\rho}+e^{-\rho^{2} / 4}\right)
$$


Lemma 6.8. There exists a constant $c$ such that

$$
\begin{aligned}
\left|\widehat{C}_{x x x}(t, x)\right| & \leq \frac{c e^{-\rho^{2} / 8}}{\rho^{2} x^{5 / 2}}(L(x, \rho)+\rho), \\
\left|\widehat{C}_{x x x x}(t, x)\right| & \leq c e^{-\rho^{2} / 8} x^{-7 / 2} P_{3}\left(\rho^{-1}\right), \\
\left|\widehat{C}_{t x}(t, x)\right| & \leq \frac{c \widehat{\sigma}^{2} e^{-\frac{\rho^{2}}{8}}}{x^{1 / 2} \rho^{2}}\left(L(x, \rho)+\rho+\rho^{2}\right), \\
\left|\widehat{C}_{x x t}(t, x)\right| & \leq c \widehat{\sigma}^{2} e^{-\rho^{2} / 8} x^{-3 / 2}\left(\rho^{-1}+\rho^{-3}\right),
\end{aligned}
$$

where $P_{3}$ is a polynomial of the third order and

$$
L(x, \rho):=\sum_{j=1}^{p} \frac{\left|\log \left(x / K_{j}\right)\right|}{\rho} \exp \left\{-\frac{\log ^{2}\left(x / K_{j}\right)}{2 \rho^{2}}\right\} .
$$

Lemma 6.9. There exists a constant $c$ and a polynomial $Q$ of third order such that

$$
\mathbf{E} S_{t}^{m} \widehat{C}_{t x}^{2}\left(t, S_{t}\right) \leq c \widehat{\sigma}_{t}^{4} Q\left(\rho^{-1}\right) e^{-\rho^{2} / 4} .
$$

\subsection{A.1.}

We give here some necessary calculus and inequalities for the present work. Moreover, we recall some results from [2] and [3]. In particular, we can show the following in a similar way:

Lemma 6.10. We have:

$$
\begin{aligned}
\widehat{C}_{x x t}(t, x)= & \frac{\widehat{\sigma}_{t}^{2}}{2 \rho_{t}^{3} x} \int_{-\infty}^{\infty} h^{\prime}\left(x e^{\rho_{t} y+\rho_{t}^{2} / 2}\right) P_{1}\left(\rho_{t}, y\right) \varphi(y) d y \\
\widehat{C}_{x t t}(t, x)= & -\frac{\rho_{t}^{\prime \prime}}{\rho_{t}} \int_{-\infty}^{\infty} h^{\prime}\left(x e^{\rho_{t} y+\rho_{t}^{2} / 2}\right) P_{2}\left(\rho_{t}, y\right) \varphi(y) d y \\
& +\frac{\widehat{\sigma}_{t}^{4}}{2 \rho_{t}^{4}} \int_{-\infty}^{\infty} h^{\prime}\left(x e^{\rho_{t} y+\rho_{t}^{2} / 2}\right) P_{3}\left(\rho_{t}, y\right) \varphi(y) d y \\
\widehat{C}_{x x x t}(t, x)= & \frac{\widehat{\sigma}_{t}^{2}}{2 \rho_{t}^{4} x^{2}} \int_{-\infty}^{\infty} h^{\prime}\left(x e^{\rho_{t} y+\rho_{t}^{2} / 2}\right) P_{4}\left(\rho_{t}, y\right) \varphi(y) d y
\end{aligned}
$$

where

$$
\begin{aligned}
P_{1}(x, y) & :=-y^{3}-x y^{2}+3 y+x \\
P_{2}(x, y) & :=-y^{2}-x y+1 \\
P_{3}(x, y) & :=y^{4}-\left(4+x^{2}\right) y^{2}+2 x y+x^{2}+1 \\
P_{4}(x, y) & :=-y^{4}+2 x y^{3}+\left(6-x^{2}\right) y^{2}-8 x y+x^{2}-3 .
\end{aligned}
$$

Moreover, we have the following inequalities: 


\section{Lemma 6.11.}

$$
\begin{aligned}
& \left|\widehat{C}_{x x t}(t, x)\right| \leq \quad c \frac{e^{-\rho_{t}^{2} / 8}}{x^{3 / 2}} \frac{\widehat{\sigma}_{t}^{2}}{\rho_{t}^{3}}\left(\sum_{j=1}^{p}\left(\varrho_{j}(x)^{2}+\rho_{t}^{2} / 4+1\right) e^{-\varrho_{j}(x)^{2} / 2}+\rho_{t}+\rho_{t}^{3}\right), \\
& \left|\widehat{C}_{x t t}(t, x)\right| \leq \quad \mathcal{X}^{1}(t, x)+\mathcal{X}^{2}(t, x)
\end{aligned}
$$

where

$$
\begin{aligned}
\mathcal{X}^{1}(t, x) & :=c \frac{e^{-\rho_{t}^{2} / 8}}{\sqrt{x}} \frac{\left|\rho_{t}^{\prime \prime}\right|}{\rho_{t}}\left(\sum_{j=1}^{p} \varrho_{j}(x) e^{-\varrho_{j}(x)^{2} / 2}+\rho_{t}+\rho_{t}^{2}\right) \\
\mathcal{X}^{2}(t, x) & :=c \frac{e^{-\rho_{t}^{2} / 8}}{\sqrt{x}} \frac{\widehat{\sigma}_{t}^{4}}{\rho_{t}^{4}}\left(\sum_{j=1}^{p}\left(\varrho_{j}(x)^{3}+\varrho_{j}(x)\right) e^{-\varrho_{j}(x)^{2} / 2}+\sum_{j=1}^{4} \rho_{t}^{j}\right)
\end{aligned}
$$

and $\varrho_{j}(x):=\left|\log \left(K_{j} / x\right)\right| / \rho_{t}$.

Lemma 6.12. Assume that Assumption A1 holds. Then there exists a constant c such that $\varepsilon_{i}:=n^{1 / 2} \sqrt{\Delta t_{i}} \sqrt{f^{\prime}\left(t_{i-1}\right)}, i \leq n-1$ satisfies the inequality $\left|\varepsilon_{i}-1\right| \leq$ $c \Delta t_{i} /\left(1-t_{i}\right)$ for $n$ large enough.

Proof. We have obviously

$$
\left|\varepsilon_{i}-1\right| \leq\left|n \Delta t_{i} f^{\prime}\left(t_{i-1}\right)-1\right|,
$$

where $\Delta t_{i}=g^{\prime}\left(\theta_{i}\right) n^{-1}$ and $\theta_{i} \in[(i-1) / n, i / n]$. Then, $d_{i}:=g\left(\theta_{i}\right)-t_{i-1} \in\left[0, \Delta t_{i}\right]$. We deduce that:

$$
\left|\varepsilon_{i}-1\right| \leq\left|\frac{f^{\prime}\left(g\left(\theta_{i}\right)-h_{i}\right)}{f^{\prime}\left(g\left(\theta_{i}\right)\right)}-1\right| \leq c \frac{\Delta t_{i}}{1-t_{i}} .
$$

Indeed, we use a first order Taylor expansion to estimate the difference $f^{\prime}\left(g\left(\theta_{i}\right)-\right.$ $\left.h_{i}\right)-f^{\prime}\left(g\left(\theta_{i}\right)\right)$. We conclude by using the explicit expression of $f, g$ but also the inequality $\left(1-t_{i-1}\right) /\left(1-t_{i}\right) \leq c$ for $i \leq n-1$.

The following lemma is of first importance to get estimations of expectations we need in some of our proofs.

Lemma 6.13. Suppose that $t \leq u<1, m \in \mathbb{R}, q \in 2 \mathbb{N}$ and $K>0$. There exists a constant $c=c(m, q)$ such that

$$
\mathbf{E} S_{u}^{m} \log ^{q} \frac{S_{u}}{K} \exp \left\{-\frac{\log ^{2}\left(S_{u} / K\right)}{\rho_{t}^{2}}\right\} \leq c P_{q}\left(\rho_{t}\right)
$$


where

$$
\begin{aligned}
P_{0}\left(\rho_{t}\right) & :=\rho_{t} \\
P_{2}\left(\rho_{t}\right) & :=\rho_{t}^{3}+\rho_{t}^{5} \\
P_{4}\left(\rho_{t}\right) & :=\rho_{t}^{5}+\rho_{t}^{7}+\rho_{t}^{9} \\
P_{2 q}\left(\rho_{t}\right) & :=\rho_{t}^{2 q+1}+\rho_{t}^{2 q+3}+\cdots+\rho_{t}^{4 q+1} .
\end{aligned}
$$

Proof. We set $p=\log \frac{S_{0}}{K}-\sigma^{2} u / 2, \alpha=\sigma \sqrt{u}$ and

$$
A(q)=\mathbf{E} S_{u}^{m} \log ^{q} \frac{S_{u}}{K} \exp \left\{-\frac{\log ^{2}\left(S_{u} / K\right)}{\rho_{t}^{2}}\right\} .
$$

Then,

$$
\begin{aligned}
& A(q)=\frac{S_{0}^{m}}{\sqrt{2 \pi}} \int_{-\infty}^{\infty}(p+\alpha y)^{q} \exp \left\{\alpha m y-\alpha^{2} m / 2-\frac{1}{\rho_{t}^{2}}(p+\alpha y)^{2}-y^{2} / 2\right\} d y \\
& A(q)=\frac{S_{0}^{m} e^{A_{1}}}{\sqrt{2 \pi}} \int_{-\infty}^{\infty}(p+\alpha y)^{q} \exp \left\{-\frac{1}{2}\left(1+\frac{2 \alpha^{2}}{\rho_{t}^{2}}\right) y^{2}+\alpha\left(m-\frac{2 p}{\rho_{t}^{2}}\right) y\right\} d y
\end{aligned}
$$

where

$$
A_{1}=-\frac{\alpha^{2} m}{2}-\frac{p^{2}}{\rho_{t}^{2}}
$$

Let $y=z / A_{2}$ with $A_{2}=\sqrt{1+2 \alpha^{2} / \rho_{t}^{2}}$. Then

$$
A(q)=\frac{S_{0}^{m} e^{A_{4}}}{\sqrt{2 \pi} A_{2}} \int_{-\infty}^{\infty}\left(p+\frac{\alpha z}{A_{2}}\right)^{q} \exp \left\{-\frac{1}{2}\left[z^{2}-2\left(A_{3} / A_{2}\right) z+A_{3}^{2} / A_{2}^{2}\right]\right\} d z
$$

where $A_{3}=\alpha\left(m-2 p / \rho_{t}^{2}\right)$ and $A_{4}=A_{1}+A_{3}^{2} /\left(2 A_{2}^{2}\right)$. After the change of variable $y=z-A_{3} / A_{2}$, we obtain that

$$
A(2)=\frac{S_{0}^{m} \rho_{t} e^{A_{4}}}{\sqrt{\rho_{t}^{2}+2 \alpha^{2}}}\left[\left(p+\frac{\alpha \rho_{t}^{2} A_{3}}{\rho_{t}^{2}+2 \alpha^{2}}\right)^{2}+\frac{\alpha^{2} \rho_{t}^{2}}{\rho_{t}^{2}+2 \alpha^{2}}\right] .
$$

Moreover, if $u \geq t$, then $\rho_{t}^{2} \geq \sigma^{2}(1-t)$ implies that

$$
\rho_{t}^{2}+2 \alpha^{2} \geq \sigma^{2}(1-t)+\sigma^{2} u \geq \sigma^{2} .
$$

We have

$$
A_{4}=-\frac{m \alpha^{2}}{2}-\frac{p^{2}}{\rho_{t}^{2}}+\frac{\alpha^{2} \rho_{t}^{2}}{2\left(\rho_{t}^{2}+2 \alpha^{2}\right)}\left(m^{2}+\frac{4 p^{2}}{\rho_{t}^{4}}-\frac{4 p m}{\rho_{t}^{2}}\right)
$$

where $p, \alpha$ are bounded. But, the term

$$
\frac{\alpha^{2} \rho_{t}^{2}}{2\left(\rho_{t}^{2}+2 \alpha^{2}\right)} m^{2}
$$

is obviously bounded whereas we can establish the following inequality

The following term

$$
\frac{\alpha^{2} \rho_{t}^{2}}{2\left(\rho_{t}^{2}+2 \alpha^{2}\right)} \frac{4 p^{2}}{\rho_{t}^{4}} \leq \frac{p^{2}}{\rho_{t}^{2}} \text {. }
$$

$$
\left|\frac{\alpha^{2} \rho_{t}^{2}}{2\left(\rho_{t}^{2}+2 \alpha^{2}\right)} \frac{4 p m}{\rho_{t}^{2}}\right|
$$


is also bounded. It follows that $e^{A_{4}}$ is bounded and we can conclude easily for $q=2$. In a similar way, we can conclude for any $q \in 2 \mathbb{N}$ because we use in particular the property

$$
\int_{-\infty}^{\infty} y^{k} \varphi(y) d y=0
$$

if $k \in 2 \mathbb{N}+1$.

From now on, we can deduce the following results.

Corollary 6.14. If $m \in \mathbb{R}$ and $u \geq t$, then there exists a constant $c_{m}>0$ such that

$$
\mathbf{E} S_{u}^{m} \widehat{C}_{x t}^{2}\left(t, S_{u}\right) \leq \frac{c_{m} \widehat{\sigma}_{t}^{4}}{\rho_{t}^{3}} e^{-\rho_{t}^{2} / 8} .
$$

Proof. Indeed, it suffices to use Lemma 6.8 and apply the previous lemma.

In a similar way, we have:

Corollary 6.15. If $m \in \mathbb{R}$ and $u \geq t$, then there exists a constant $C_{m}>0$ such that

$$
\begin{aligned}
\mathbf{E} S_{u}^{m} \widehat{C}_{x t}^{4}\left(t, S_{u}\right) & \leq \frac{c_{m} \widehat{\sigma}_{t}^{8}}{\rho_{t}^{7}} e^{-\rho_{t}^{2} / 8} \\
\mathbf{E} S_{u}^{m} \widehat{C}_{x x}^{4}\left(t, S_{u}\right) & \leq \frac{c_{m}}{\rho_{t}^{3}} e^{-\rho_{t}^{2} / 4} .
\end{aligned}
$$

Corollary 6.16. If $m \in \mathbb{R}$ then there exists a constant $c_{m}>0$ such that

$$
\mathbf{E} S_{t}^{m} \widehat{C}_{x x}^{5 / 2}\left(t, S_{t}\right) \leq \frac{c_{m}}{\rho_{t}^{15 / 8}} e^{-5 \rho_{t}^{2} / 32} .
$$

Proof. We write

$$
\mathbf{E} S_{t}^{m} \widehat{C}_{x x}^{5 / 2}\left(t, S_{t}\right)=\mathbf{E} S_{t}^{m} \widehat{C}_{x x}^{3 / 2}\left(t, S_{t}\right) \widehat{C}_{x x}\left(t, S_{t}\right)
$$

and we apply Cauchy-Schwarz' inequality with $p=4 / 3$ and $q=4$ such that $p^{-1}+$ $q^{-1}=1$. We obtain

$$
\begin{aligned}
\mathbf{E} S_{t}^{m} \widehat{C}_{x x}^{5 / 2}\left(t, S_{t}\right) & \leq\left(\mathbf{E} S_{t}^{4 m / 3} \widehat{C}_{x x}^{2}\left(t, S_{t}\right)\right)^{3 / 4}\left(\mathbf{E} \widehat{C}_{x x}^{4}\left(t, S_{t}\right)\right)^{1 / 4} \\
& \leq\left(C_{m} \mathbf{E} \widehat{C}_{x x}^{4}\left(t, S_{t}\right)\right)^{3 / 8}\left(\mathbf{E} \widehat{C}_{x x}^{4}\left(t, S_{t}\right)\right)^{1 / 4} \\
& \leq\left(C_{m} \frac{c}{\rho_{t}^{3}} e^{-\rho_{t}^{2} / 4}\right)^{3 / 8}\left(\frac{c}{\rho_{t}^{3}} e^{-\rho_{t}^{2} / 4}\right)^{1 / 4}
\end{aligned}
$$

where the last inequality is deduced from Corollary 6.119. The conclusion follows. 
Corollary 6.17. If $m \in \mathbb{R}$ and $u \geq t$, then there exists a constant $c_{m}>0$ such that

$$
\begin{aligned}
\mathbf{E} S_{u}^{m} \widehat{C}_{x x x}^{2}\left(t, S_{u}\right) & \leq \frac{c_{m}}{\rho_{t}^{3}} e^{-\rho_{t}^{2} / 8} \\
\mathbf{E} S_{u}^{m} \widehat{C}_{x x t}^{2}\left(t, S_{u}\right) & \leq \frac{c_{m} \widehat{\sigma}_{t}^{4}}{\rho_{t}^{5}} e^{-\rho_{t}^{2} / 8} \\
\mathbf{E} S_{u}^{m} \widehat{C}_{x x x}^{4}\left(t, S_{u}\right) & \leq \frac{c_{m}}{\rho_{t}^{7}} e^{-\rho_{t}^{2} / 8} \\
\mathbf{E} S_{u}^{m} \widehat{C}_{x x x x}^{2}\left(t, S_{u}\right) & \leq \frac{c_{m}}{\rho_{t}^{5}} e^{-\rho_{t}^{2} / 8} \\
\mathbf{E} S_{u}^{m} \widehat{C}_{x x t}^{4}\left(t, S_{u}\right) & \leq \frac{c_{m} \widehat{\sigma}_{t}^{8}}{\rho_{u}^{11}} e^{-\rho_{t}^{2} / 8} .
\end{aligned}
$$

Let $\widetilde{S}_{t_{i-1}} \in\left[S_{t_{i-1}}, S_{t_{i}}\right]$ and $\widetilde{t}_{i-1} \in\left[t_{i-1}, t_{i}\right]$ be some random variables. We have the following inequalities:

Lemma 6.18. There exists a constant $c$ such that

$$
\mathbf{E} \widehat{C}_{x t}^{4}\left(\widetilde{t}_{i-1}, \widetilde{S}_{t_{i-1}}\right) \leq \frac{c e^{-\rho_{t_{i}}^{2} / 4}}{\left(1-t_{i}\right)^{4}}
$$

Proof. We have $\widetilde{S}_{t_{i-1}}^{m} \leq S_{t_{i-1}}^{m}+S_{t_{i}}^{m}$, and $\rho_{\widetilde{t}_{i-1}} \geq \rho_{t_{i}}$. Furthermore, in virtue of Lemma 6.8 , recall that we have

$$
\left|\widehat{C}_{x t}(t, x)\right| \leq c \frac{\widehat{\sigma}_{t}^{2} e^{-\rho_{t}^{2} / 8}}{x^{1 / 2} \rho_{t}^{2}} .
$$

Then, the conclusion follows.

In the same way, we can prove the following results:

Lemma 6.19. There exists a constant $C$ such that

$$
\mathbf{E} \widehat{C}_{x t t}^{4}\left(\widetilde{t}_{i-1}, \widetilde{S}_{t_{i-1}}\right) \leq \frac{C e^{-\rho_{t_{i}}^{2} / 4}}{\left(1-t_{i}\right)^{8}} .
$$

Proof. The arguments are similar to the previous ones but we also use the inequality:

$$
\frac{g^{\prime \prime}(u)}{g^{\prime}(u)^{2}} \leq \frac{C}{(1-g(u))^{3 / 2}}, \forall u<1
$$

in order to have

$$
\frac{\rho_{t}^{\prime \prime}}{\rho_{t}} \leq \frac{C}{(1-t)^{2}}
$$


Lemma 6.20. There exists a constant $C$ such that

$$
\begin{aligned}
\mathbf{E} \widehat{C}_{x x x}^{4}\left(\widetilde{t}_{i-1}, \widetilde{S}_{t_{i-1}}\right) & \leq \frac{C e^{-\rho_{t_{i}}^{2} / 4}}{\rho_{t_{i}}^{8}} \\
\mathbf{E} \widehat{C}_{x x t}^{4}\left(\widetilde{t}_{i-1}, \widetilde{S}_{t_{i-1}}\right) & \leq \frac{C e^{-\rho_{t_{i}}^{2} / 4}}{n\left(1-t_{i}\right)^{6} f^{\prime}\left(t_{i}\right)} \\
\mathbf{E} \widehat{C}_{x x x x}^{4}\left(\widetilde{t}_{i-1}, \widetilde{S}_{t_{i-1}}\right) & \leq \frac{C e^{-\rho_{t_{i}}^{2} / 4}}{\rho_{t_{i}}^{12}} .
\end{aligned}
$$

\subsection{A.2.}

Recall the two following lemmas (see [2]). These results ensures the convergence of the Leland scheme without any hedging error when using the modified Leland strategy. The change of variable $x=\rho_{u}^{2}$ appears to be as essential in the following proofs and points out the significative role of the revision dates near the maturity.

Lemma 6.21. We have the following equality

$$
\int_{s}^{t} \widehat{C}_{x t}\left(u, S_{u}\right) d u=\int_{\rho_{t}^{2}}^{\rho_{s}^{2}} \widehat{C}_{x t}\left(u, S_{u}\right) \widehat{\sigma}_{u}^{-2} d x
$$

where $u=u(x, n)$ is defined by $x=\rho_{u}^{2}$ and verifies $\lim _{n \rightarrow \infty} u(x, n)=1$. Moreover,

$$
C_{x t}\left(u, S_{u}\right) \widehat{\sigma}_{u}^{-2}=\frac{1}{2 x} \int_{-\infty}^{\infty} h^{\prime}\left(S_{u} e^{\sqrt{x} y+x / 2}\right)\left(-y^{2}-\sqrt{x} y+1\right) \varphi(y) d y
$$

satisfies the following inequality

$$
\left|\widehat{C}_{x t}\left(u, S_{u}\right) \widehat{\sigma}_{u}^{-2}\right| d u \leq c G_{1}\left(x, S_{u}\right)
$$

where

$$
G_{1}(x, S):=\frac{1}{x} e^{-x / 8}\left(\sum_{j=1}^{p} \frac{\left|\log \left(S / K_{j}\right)\right|}{\sqrt{x}} \exp \left\{-\frac{\log ^{2}\left(S / K_{j}\right)}{2 x}\right\}+\sqrt{x}+x\right) .
$$

Corollary 6.22. Assume that we have two sequences $\left(t_{k^{n}}\right)_{n \in \mathbf{N}}$ and $\left(s_{k^{n}}\right)_{n \in \mathbf{N}}$ in $[0,1]$ such that $\rho_{t_{k^{n}}}$ and $\rho_{s_{k^{n}}}$ respectively converge to $a \in[0, \infty]$ and $b \in[0, \infty]$. Then,

$$
\lim _{n \rightarrow \infty} \int_{s_{k^{n}}}^{t_{k^{n}}} \widehat{C}_{x t}\left(u, S_{u}\right) d u=\int_{a}^{b} J\left(x, S_{1}\right) d x<\infty, \text { a.s. }
$$

Proof. We apply Lemma 6.21 with the change of variable $x=\rho_{u}^{2}$. Recall that $0 \leq 1-u \leq \operatorname{cxn}^{-1 / 2}$ so that $u \rightarrow 1$ as $n \rightarrow \infty$ for a given $x \geq 0$. We can apply the Lebesgue theorem by dominating the function $G_{1}\left(x, S_{u}\right)$ whether $x \leq 1$ or not because $x \leq 1$ implies that $u$ is sufficiently near from 1 independently of $x$ for $n \geq$ 
$n_{0}$. Indeed, outside of the null-set $\cup_{i}\left\{S_{1}=K_{i}\right\}$, we have $0<a \leq\left|\log \left(S_{u} / K_{j}\right)\right| \leq b$

for some constants $a, b$ (depending on $\omega$ ) provided that $u$ is sufficiently near one.

\section{REFERENCES}

[1] E. Denis. Marchés avec coûts de transaction: approximation de Leland et arbitrage. Thèse, Université de Franche-Comté, 2008.

[2] E. Denis. Modified Leland's strategy for constant transaction costs rate. Besancon, preprint 2009/19. In revision in Mathematical Finance.

[3] E. Denis. Approximate hedging of contingent claims under transaction costs. To appear in Applied Mathematical Finance.

[4] E. Denis, Y. Kabanov. Mean square error for the Leland-Lott hedging strategy: convex payoffs. Finance and Stochastics.

[5] M. Gamys, Y. Kabanov. Mean square error for the Leland-Lott hedging strategy. Recent Advances in Financial Engineering: Proceedings of the 2008 Daiwa International Workshop on Financial Engineering. World Scienific, Singapore-New Jersey-London-Hong Kong (2009)

[6] P. Granditz, W. Schachinger. Leland's approach to option pricing: The evolution of discontinuity. Mathematical Finance, 11, 347-355 (2001)

[7] P. Hall, C. C. Heyde. Martingale limit theory and its application. Probability and Mathematical Statistics. Academic Press, Inc. [Harcourt Brace Jovanovich, Publishers], New YorkLondon, 1980. xii +308 pp

[8] Y. Kabanov, M. Safarian. On Leland's strategy of option pricing with transaction costs. Finance and Stochastics, 1, 239-250 (1997)

[9] H. Leland. Option pricing and replication with transactions costs, Journal of Finance, XL, 1283-1301 (1985)

[10] K. Lott. Ein verfahren zur replikation von optionen unter transaktionkosten in stetiger zeit, Dissertation. Universität der Bundeswehr München. Institut für Mathematik und Datenverarbeitung (1993)

[11] S. Pergamenshchikov. Limit theorem for Leland's strategy. The Annals of Applied Probability, 13, 1099-1118 (2003)

[12] J. Sekine, J. Yano. Hedging errors of Leland's strategies with time-inhomogeneous rebalancing. Preprint. 